\title{
3D Bioprinting for Cartilage and Osteochondral Tissue Engineering
}

\author{
Andrew C. Daly ${ }^{1,2,3}$, Fiona F Freeman ${ }^{1,2,3}$, Tomas Fernandez Gonzalez ${ }^{1,2,3,4}$ Susan E \\ Critchley $^{1,2,3}$, Jessica Nulty ${ }^{1,2,3}$, Daniel J Kelly ${ }^{1,2,3,4}$
}

${ }^{1}$ Trinity Centre for Bioengineering, Trinity Biomedical Sciences Institute, Trinity College Dublin, Dublin, Ireland.

${ }^{2}$ Department of Mechanical and Manufacturing Engineering, School of Engineering, Trinity College Dublin, Dublin, Ireland.

${ }^{3}$ Department of Anatomy, Royal College of Surgeons in Ireland, Dublin, Ireland

${ }^{4}$ Advanced Materials and Bioengineering Research Centre (AMBER), Royal College of Surgeons in Ireland and Trinity College Dublin, Dublin, Ireland.

*Corresponding Author: Tel: +353-1-8963947, email: kellyd9@tcd.ie 


\section{Abstract}

Significant scientific progress has been made in the field of cartilage and bone tissue engineering over the last two decades. As a result, there is real promise that strategies to regenerate rather than replace damaged or diseased bones and joints will one day reach the clinic, however a number of major challenges must still be addressed before this becomes a reality. These include vascularization in the context of large bone defect repair, engineering complex gradients for bone-soft tissue interface regeneration and recapitulating the stratified zonal architecture present in many adult tissues such as articular cartilage. Tissue engineered constructs typically lack such spatial complexity in cell types and tissue organization, which may explain their relatively limited clinical success to date. This has led to increased interest in biofabrication technologies in the field of musculoskeletal tissue engineering. The additive, layer-by-layer nature of bioprinting strategies makes it possible to generate zonal distributions of cells, matrix and bioactive cues in 3D. The adoption of biofabrication technology in musculoskeletal tissue engineering may therefore make it possible to produce the next generation of biological implants capable of treating a range of conditions. Here we will review advances in bioprinting for cartilage and osteochondral tissue engineering, from the engineering of fiber reinforced tissues to biofabrication strategies for whole joint regeneration. Furthermore, we will outline some of the key future research directions for this burgeoning field, from bioprinting of vascularised constructs to the development of gene activated bioinks for interface tissue engineering. 


\section{Contents}

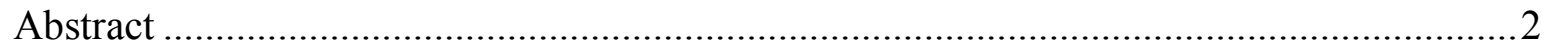

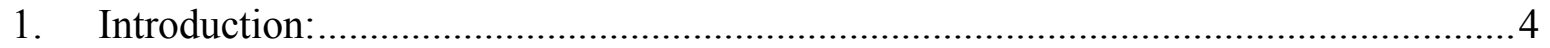

2. 3D Bioprinting in Cartilage and Bone Tissue Engineering: .......................................... 7

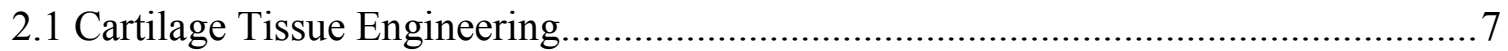

2.1.1 Bioprinting Technology for Cartilage Tissue Engineering ................................ 7

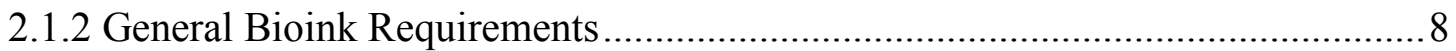

2.1.3 Bioink Development for Cartilage Tissue Engineering ..................................... 9

2.1.4 Bioprinting of Heterogeneous Cartilage Tissues .............................................. 12

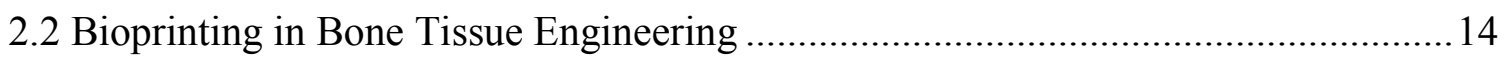

2.2.1 Developing bioinks for bone tissue engineering............................................ 14

2.2.2 Bioprinting and vascularisation in BTE........................................................ 16

2.3 Bioprinting of Composite Reinforced Tissues .......................................................... 19

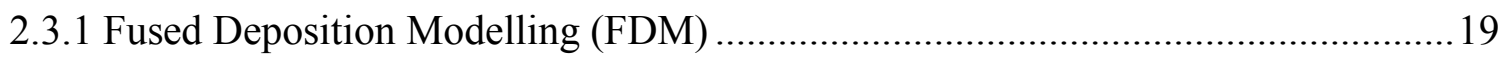

FDM in Cartilage and Osteochondral Tissue Engineering .....................................20

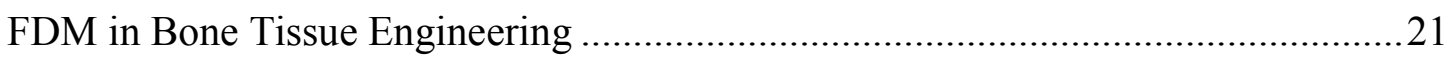

2.3.2 Bioprinting of Reinforced Constructs for Cartilage and Osteochondral Tissue

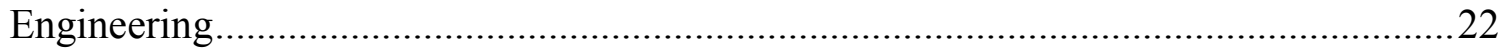

2.3.3 Bioprinting of Reinforced Constructs for Bone Tissue Engineering .....................26

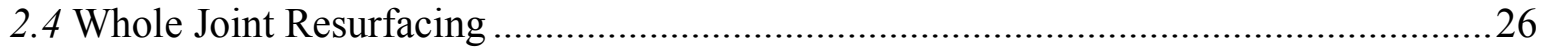

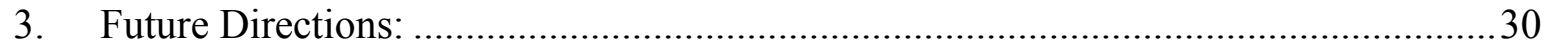

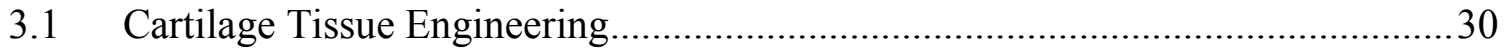

3.1.1 Biofabrication of Mechanically Functional Cartilage Tissues............................30

3.1.2 Bioprinting of Stratified Cartilage Tissues and Osteochondral Tissue Interfaces

3.1.3 Towards Biofabrication of Anatomically Accurate Cartilage Tissues............33

3.2 Bioprinting of Vascularised Bone Tissue Engineering Constructs .............................34

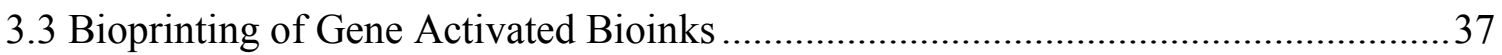

3.3.1 Engineering Zonally Organised Interface Tissues Using Nucleic Acid Delivery

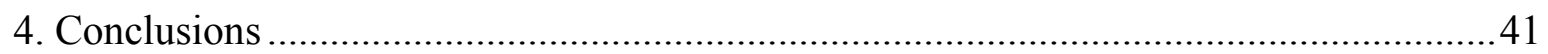

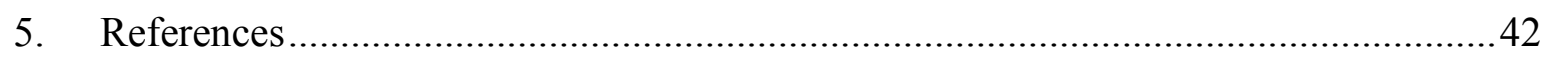




\section{Introduction:}

Osteoarthritis (OA) is a degenerative joint disease that affects millions of people worldwide. In the USA alone, OA affects $37 \%$ of adults over 65 years old ${ }^{1}$. The disease is characterised by progressive loss of hyaline cartilage in the synovial joints which leads to significant joint pain, swelling and stiffness for sufferers. The disease is also a significant economic burden with associated costs estimated to range from \$3.4-13.2 billion per year in the USA ${ }^{2}$. The current gold standard treatment option for OA is total joint arthroplasty where the diseased cartilage and underlying bone are replaced with a metal and polymer prosthesis. While the procedure is well established failures and complications are not uncommon ${ }^{3,4}$. For example, ten year revision rates of up to $12 \%$ have been reported ${ }^{5}$. This has led to an increased interest in the field of cartilage and osteochondral tissue engineering (TE) where de novo tissues can be engineered to facilitate joint regeneration and hopefully prevent the onset of OA.

Significant progress has been made in the field of TE over the last two decades with numerous studies demonstrating how combinations of biomaterials, cells and bioactive factors can be used to engineer de novo cartilage and bone in vitro and in vivo ${ }^{6-11}$. In the case of cartilage tissue engineering this has classically involved encapsulating chondrocytes, or stem cells which can be differentiated along a chondrogenic linage, in a supportive matrix such as a hydrogel or scaffold. The efficacy of such approaches for treating focal cartilage or osteochondral defects have been demonstrated by a number of groups in large animal models 12-18. In addition, a number of chondrocyte based therapies such as MACI (autologous cultured chondrocytes on porcine collagen membrane) are available clinically and newer tissue engineered cartilage products have entered the clinical trial stage, with some demonstrating improvements in defect healing compared to existing treatment options such as microfracture 
or autologous chondrocyte implantation ${ }^{19}$. However many products have also failed to demonstrate efficacy and challenges remain in translating TE technologies into the clinic ${ }^{19}$. Furthermore, existing approaches are designed to repair focal cartilage defects, but are not suitable for treating osteoarthritic joints. The majority of TE products are typically formed using mechanically weak hydrogels or scaffolds and are not suitable for treating the large areas of degenerative joint surfaces associated with diseases such as OA. It is evident that a new generation of more sophisticated tissue engineered cartilage and osteochondral grafts are required to treat this more challenging patient population.

TE strategies typically aim to homogenously distribute biological factors such as cells and growth factors throughout a biomaterial matrix. As a result, engineered tissues are often homogenous in composition. However, articular cartilage is a highly anisotropic tissue whose composition and organization varies greatly with depth. The tissue can be divided into three zones, the superficial, middle and deep zone which are defined by gradients in collagen and proteoglycan content and collagen fiber alignment ${ }^{20-26}$. These variations in ECM composition and architecture in turn impart zonal biomechanical properties to the tissue ${ }^{24,27}$. It is generally accepted that further progress in the field will require strategies that can better recapitulate the spatial complexity of the native tissue and its interface with subchondral bone ${ }^{28}$. The next generation of cartilage and osteochondral tissue engineered products should therefore incorporate these considerations. Another major challenge in the field of cartilage TE is that the mechanical properties of tissue engineered cartilage often fall below those of the native tissue. Ideally tissue engineered cartilage would be able to withstand the high levels of compressive and shear loading that will be present in an articulating joint upon implantation. This is another key consideration for the next generation of tissue engineered cartilage products, especially those designed to treat joints with large areas of degeneration. 
The aforementioned issues have led to an increased interest in the use of biofabrication for cartilage and osteochondral tissue engineering as the additive, layer-by-layer technology makes it possible to spatially pattern cells, bioactive factors and biomaterials in $3 \mathrm{D}^{29-31}$. For example, polymer reinforced hydrogels with mechanical properties approaching those of native cartilage can be printed using multi-tool biofabrication ${ }^{32,33}$. In addition bioprinting technology can be used to engineer zonally organised constructs with gradients of cells and biological cues ${ }^{34}$. In this review we will discuss developments in bioprinting for cartilage tissue engineering. In addition, since damaged or diseased articular cartilage is also commonly associated with defects or degeneration of the underlying subchondral bone, we will also review developments in bioprinting for bone tissue engineering and how the technology can be used to engineer osteochondral implants. Finally we will discuss some potential future directions for the field and how biofabrication technologies could be used to develop the next generation of cartilage and osteochondral grafts suitable for treating challenging joint defects and potentially osteoarthritic joints. 


\section{3D Bioprinting in Cartilage and Bone Tissue Engineering:}

\subsection{Cartilage Tissue Engineering (CTE)}

\subsubsection{Bioprinting Technology for CTE}

A number of groups have started to explore the use of microextrusion and inkjet based bioprinting of cell laden hydrogels for CTE. Microextrusion is a widely used technique where bioink strands are extruded through a nozzle, onto a substrate, from a pressurised syringe barrel. Hydrogels are mainly used as the ink since they can be extruded while supporting a cargo of viable cells, growth factors and/or genetic material. The viscosity of the bioink must be sufficiently high to avoid tension driven droplet formation at the nozzle tip for extrusion of continues filaments. In an early approach, chondrocytes were extruded into simple geometries using a hyaluronic acid and dextran based hydroge ${ }^{35}$. The authors observed high cell viability for up to 3 days in vitro demonstrating the promise of such approaches. In another early approach, chondrocytes were encapsulated in gelatin metacrylamide (GelMA) hydrogels and extruded into simple porous grid structures for CTE ${ }^{36}$. The cells were cultured for 4 weeks in vitro and the author's demonstrated sGAG and collagen type II production within the constructs. Droplet based bioprinting has also been explored for CTE. Droplet bioprinting systems deposit discrete volumes of bioink during translation rather than continuous strands, like in microextrusion. These droplets can be generated by using either inkjet ${ }^{37-40}$, micro-valve 41,42 or acoustic droplet ${ }^{43-45}$ technology. In an early proof-of-principal approach, human articular chondrocytes were encapsulated in a poly(ethylene) glycol dimethacrylate (PEGDMA) bioink and inkjet printed to create 3D tissue constructs (Fig 1a) ${ }^{46}$. This study demonstrated that chondrocytes were viable and capable of synthesising cartilage matrix components post-printing. In another study, the same authors demonstrated how it was possible 
to leverage the process to directly print into a cartilage defect ${ }^{47}$. Matrix formation was assessed after six weeks and despite a visible interface between the native and repaired cartilage, the interface failure stress had significantly increased, demonstrating the potential of this approach. For more detailed information on microextrusion and inkjet bioprinting the reader is directed to a number of excellent reviews on this topic $31,48,49$.

\subsubsection{General Bioink Requirements}

A wide range of bioinks, with suitable rheological behaviour, have been developed for microextrusion and inkjet bioprinting. For example, alginate ${ }^{50-53}$, GelMA ${ }^{54,55}$, agarose ${ }^{56,57}$, collagen ${ }^{58,59}$, fibrin ${ }^{60-62}$, silk ${ }^{63-65}$, forms of poly(ethylene) glycol (PEG) ${ }^{66-68}$ and hyaluronic acid ${ }^{69,70}$ have all been shown to be compatible with such bioprinting technology. A number of criteria must be met when developing bioinks for microextrusion. First, the bioink must have suitable rheological properties for controlled microextrusion, and second, it must also be capable of supporting cell growth and tissue development post-printing. Developing bioinks that can satisfy these two requirements is challenging. Cells benefit from lower polymer concentrations and cross linking densities where they can more readily differentiate and proliferate toward a target tissue ${ }^{71-73}$. However higher polymer concentrations often result in hydrogels with suitable rheological behaviour for extrusion as the viscosity is increased. In addition, higher polymer concentrations are typically associated with improved mechanical properties. These opposing requirements form what has been coined as the biofabrication window ${ }^{74}$. The most important rheological parameters to consider when designing bioinks are viscosity, yield stress and shear thinning behaviour ${ }^{74}$. Higher polymer densities are more suitable for microextrusion since they are more viscous and possess a higher yield stress.

However it should be noted that higher viscosity inks, along with higher extrusion pressures and smaller diameter nozzles, increase shear forces experienced by cells during 
extrusion, which can lead to cell death ${ }^{75}$. For example, shear stresses greater than $60 \mathrm{kPa}$ have been shown to kill greater than $35 \%$ of cells during microextrusion ${ }^{77}$. As a result, the resolution of microextrusion based bioprinting is limited by shear induced cell death. Typically filament diameters of $150-2000 \mu \mathrm{m}$ can be achieved by controlling the bioink viscosity, extrusion pressure and nozzle diameter ${ }^{54,76,78}$. The lowest resolution nozzle typically used for microextrusion of cells is 30 Gauge $(159 \mu \mathrm{m}$ diameter). The needle geometry can also be controlled to reduce shear forces experienced by cells during extrusion ${ }^{54}$. For example, at lower inlet pressures cell viability can be increased by using conical rather than cylindrical needles. Interestingly however, at higher inlet pressures conical needles supported better cell viability. The authors hypothesised that both the magnitude of shear stress along with the exposure time to the shear stress are important. For more information of bioink development the reader is directed to a number of excellent reviews ${ }^{74,79,80}$.

\subsubsection{Bioink Development for CTE}

A number of different bioinks have been explored for CTE. In an early study, it was demonstrated how bioprinting technology could be used to engineer osteochondral tissue constructs using an alginate bioink ${ }^{81}$. Bi-layered implants were printed with chondrocytes encapsulated in the cartilage layer and osteogenic progenitors encapsulated in the bone layer (Fig 1b). Distinctive tissue formation was observed both in vitro and in vivo using this system. Cartilage matrix components such as hyaluronic acid and chondroitin sulphate can be combined with alginate to create more biomimetic hydrogels capable of supporting superior neocartilage formation ${ }^{82}$. GelMA has also been shown to support cartilage tissue formation using chondrocytes and MSCs ${ }^{36}$. However, GelMA typically supports the development of a more fibrocartilaginous type tissue with higher levels of collagen type I production ${ }^{83}$. Again however, the addition of hyaluronic acid and chondroitin sulphate to GelMA can enhance chondrogenesis ${ }^{36,84}$. In addition, the incorporation of these components has the added benefit 
of increasing the bioink viscosity which can enhance printability ${ }^{36}$. In a similar approach, a synthetic thermosensitive hydrogel composed of a base methacrylated polyHPMA-lac-PEG triblock copolymer was chemically combined with either methacrylated chondroitin sulphate (CSMA) or methacrylated hyaluronic acid (HAMA) for cartilage bioprinting ${ }^{85}$.

Novel shear thinning bioinks have also been developed for CTE by combining alginate and nanofibrillated cellulose ${ }^{86}$. These inks are highly printable, capable of supporting cell proliferation and the subsequent synthesis of cartilage matrix components ${ }^{86,87}$. The chondrogenic capacity of these inks can be enhanced by sulphating the alginate component ${ }^{87}$. The sulfation of the alginate has been shown to maintain the phenotype of chondrocytes through activation of FGF (Fibroblastic Growth Factor) signalling ${ }^{88}$. However, care must be taken when utilizing nanocellulose as a thickening agent. The resultant inks are highly viscous and it has been demonstrated that after extrusion through smaller diameter nozzles, excess shear stress can cause chondrocytes to lose their capacity proliferate and synthesis ECM components ${ }^{87}$.

Recently, a number of groups have started to explore the use of extracellular matrix (ECM) based bioinks ${ }^{89-92}$. It is believed that the incorporation of tissue specific ECM components into a bioink can provide environments more conducive to supporting specific cellular phenotypes. In one example, heart, fat and cartilage tissue were first decellularised and then solubilised at neutral $\mathrm{pH}$ at $4^{\circ} \mathrm{C}$ to create an extrudable thermosensitive bioink ${ }^{89}$. The solubilised ECM bioink could be stored at $4^{\circ} \mathrm{C}$ in an extrudable form. Post-printing, the bioink filaments could be solidified by raising the temperature to $37^{\circ} \mathrm{C}$. It was found that the encapsulated cells remained viable post-printing and were capable of differentiating toward the lineage specific to the ECM used to form the ink. It was also possible to deposit the bioink within a polymeric framework to generate three dimensional tissue templates. An identical approach has been used by the same authors using a skeletal muscle derived bioink further 
demonstrating the versatility of the approach ${ }^{90}$. In another study, a chondroinductive bioink was developed by combining gellan, alginate and cartilage ECM particles ${ }^{91}$. Highly accurate anatomical shapes could be printed with the ink and chondrocytes were capable of proliferating and producing matrix components within it (Fig 1c). In a novel scaffold-free bioprinting approach, modular cartilage tissue strands, which were fabricated by fusing tissue spheroids in a confining mold, were capable of being printed into 3D constructs using a robotic dispensing system ${ }^{93}$. A summary of bioinks used in CTE to date is provided in table 1 below.

\section{Table 1: Summary of bioinks used for CTE}

Scoring system,

Chondrogenic capacity $=1$, defines a material that has no known inherent chondroinductive factors. Chondrogenic capacity $=2$, defines material that can support chondrogenesis of MSCs without the addition of exogenous growth factors such as TGF- 33 .

Inherent printability $=1$, defines a material that cannot be extruded in a reliable fashion for more than 1 layer with ease. Inherent printability $=2$, defines a material that can be extruded into relatively simple shapes. Inherent printability $=\mathbf{3}$, defines a material that can be extruded into complex shapes with overhanging structures

\begin{tabular}{|c|c|c|}
\hline Bioink & $\begin{array}{l}\text { Inherent chondrogenic } \\
\text { capacity }\end{array}$ & Inherent printability \\
\hline Agarose $^{83}$ & 1 & $\begin{array}{l}1 \text { (Challenging to print high aspect ratios without } \\
\text { supporting structures) }\end{array}$ \\
\hline Alginate $^{83,94}$ & 1 & $\begin{array}{l}2 \text { (requires smart cross-linking approaches/thickening } \\
\text { agents to print higher shape fidelity constructs) } \\
50,94,95\end{array}$ \\
\hline Sulphated Alginate ${ }^{88}$ & $\begin{array}{l}2 \text { (can bind growth factors such } \\
\text { as fibroblast growth factor } \\
\text { (FGF), transforming growth } \\
\text { factor (TGF) and induces potent } \\
\text { proliferation and collagen II } \\
\text { deposition by encapsulated } \\
\text { bovine chondrocytes }\end{array}$ & $\begin{array}{l}2 \text { (requires smart cross-linking approaches to print higher } \\
\text { shape fidelity constructs) }\end{array}$ \\
\hline Alginate/Nano-cellulose ${ }^{87}$ & $\begin{array}{l}1 \text { (effect of the addition of } \\
\text { nanocellulose not studied) }\end{array}$ & 3 (nanocellulose imparts shear thinning behaviour) \\
\hline GelMA $^{83,96}$ & 1 & $\begin{array}{l}2 \text { (requires smart cross-linking approaches to print higher } \\
\text { shape fidelity constructs) }\end{array}$ \\
\hline Hyaluronic Acid ${ }^{36,82,97}$ & 2 & 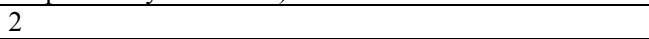 \\
\hline GelMA + (hyaluronic acid) ${ }^{36}$ & $\begin{array}{l}2 \text { (Addition of HA enhances } \\
\text { chondrogenesis) }\end{array}$ & 2 (Addition of HA enhances rheological behaviour) \\
\hline GelMA/Gellan Gum ${ }^{78,96}$ & $\begin{array}{l}1 \text { (Addition of gellan gum does } \\
\text { not enhance chondrogenesis } \\
\text { compared to GelMA alone) }\end{array}$ & $\begin{array}{l}3 \text { (addition of gellan gum imparts shear thinning } \\
\text { behaviour) }\end{array}$ \\
\hline $\begin{array}{l}\text { PEGDMA (poly(ethylene) glycol } \\
\text { dimethacrylate) }\end{array}$ & 1 & $\begin{array}{l}2 \text { (requires smart cross-linking approaches/thickening } \\
\text { agents to print high shape fidelity constructs) }{ }^{69}\end{array}$ \\
\hline Collagen $^{58}$ & 1 & $\begin{array}{l}2 \text { (Requires high collagen densities for controlled } \\
\text { extrusion } \sim 15 \mathrm{mg} / \mathrm{ml} \text { ) }\end{array}$ \\
\hline Fibrin ${ }^{98}$ & 1 & $\begin{array}{l}1 \text { (requires smart cross-linking approaches/thickening } \\
\text { agents to print higher shape fidelity constructs) }\end{array}$ \\
\hline $\begin{array}{l}\text { Methacrylated Poly[N-(2-hydroxypropyl) } \\
\text { methacrylamide mono/dilactate]-PEG } \\
\text { Triblock } 85\end{array}$ & $\begin{array}{l}2 \text { (Incorporates methacrylated } \\
\text { chondroitin sulphate (CSMA) or } \\
\text { methacrylated hyaluronic acid } \\
\text { (HAMA) to support } \\
\text { chondrogenesis }\end{array}$ & 2 \\
\hline
\end{tabular}




\subsubsection{Bioprinting of Heterogeneous Cartilage Tissues}

Recently a number of studies have explored whether bioprinting can be used to engineer cartilage tissues with regional distinctions in their composition. Spatially heterogeneous, anatomically shaped constructs have been bioprinted using a high density collagen bioink for $\mathrm{CTE}^{58}$. The constructs were able to support cell growth and it was possible to spatially localise two different cell populations. In addition, it was also possible to modulate the regional mechanical properties of the construct by varying the bioink density. This is an important consideration for $\mathrm{CTE}$ as the stiffness of the underlying substrate can influence the chondrogenic capacity of MSCs, with stiffer matrices supporting a more transient hypertrophic

phenotype ${ }^{72}$. As mentioned previously, the composition of articular cartilage varies greatly with depth. In an attempt to recapitulate this structure, Ren et al (2016) bioprinted a density gradient of chondrocytes in a single construct using a collagen type II bioink ${ }^{34}$. The cell density gradient resulted in a graded distribution of ECM components, demonstrating the promise of such biomimetic approaches. A wide range of alternative approaches could be explored in order to engineer zonally organised cartilage tissues using bioprinting technology. These will be further discussed in section 3.1.2. 
A

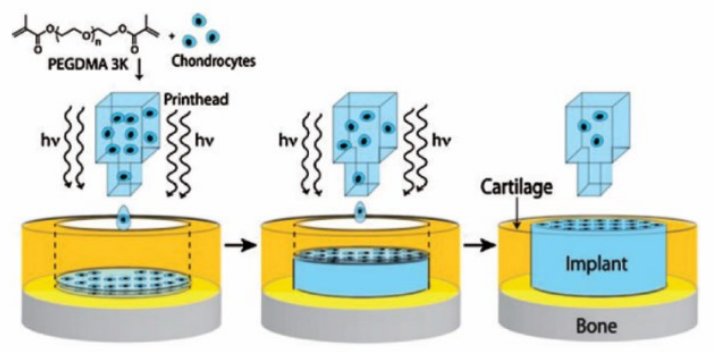

B

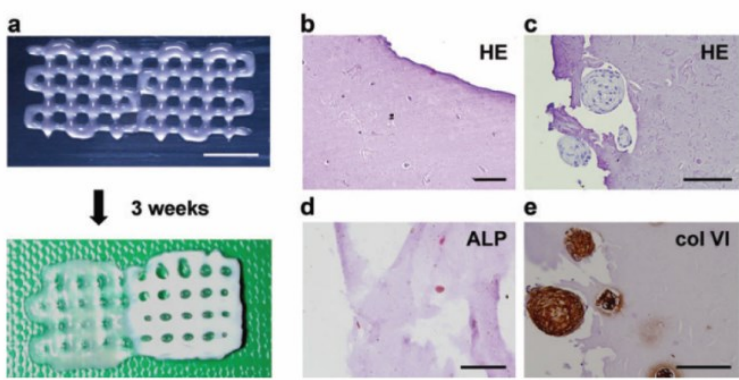

C
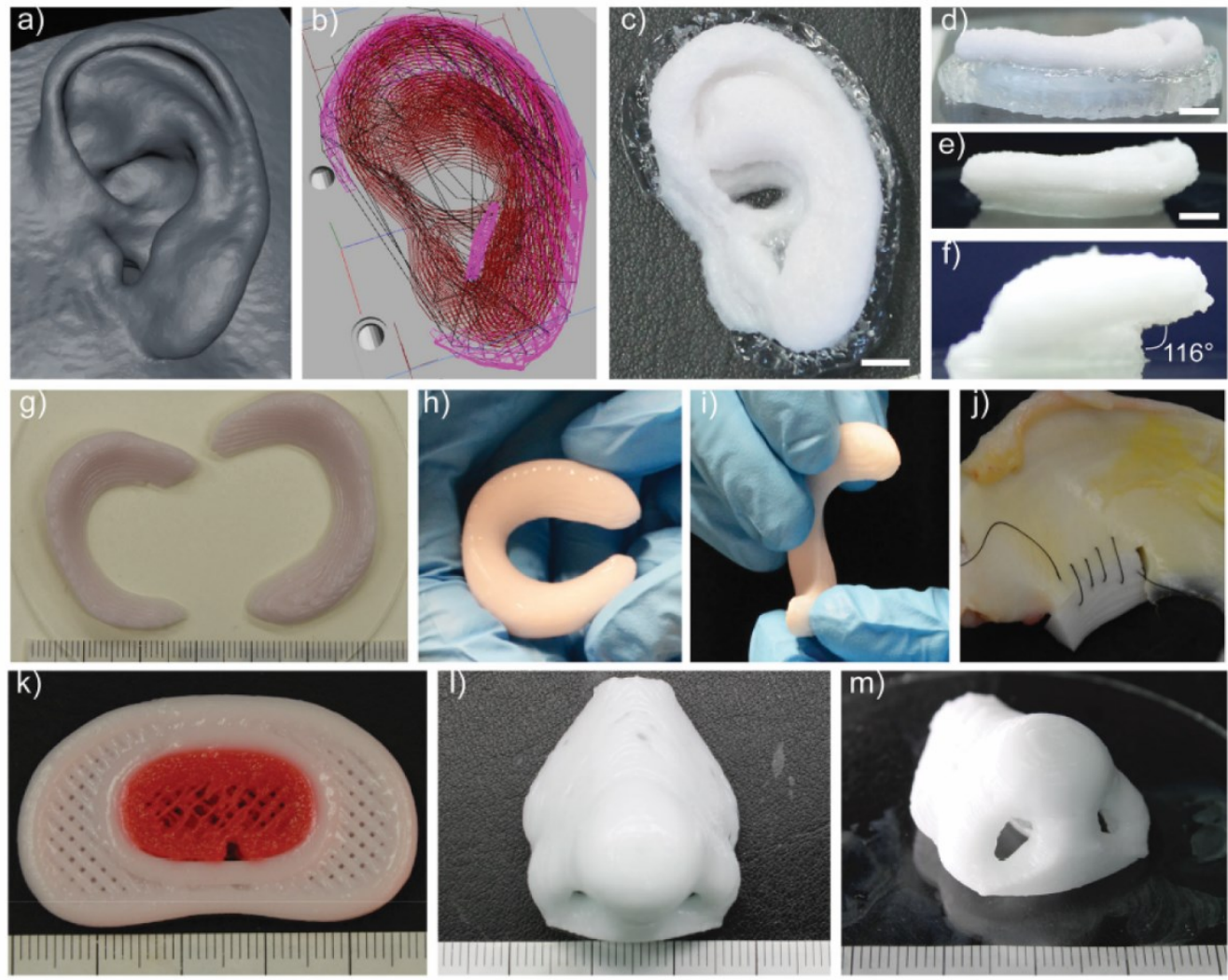

Figure 1: Applications of biofabrication technology in cartilage tissue engineering. (A)

Direct inkjet bioprinting of chondrocytes loaded in a photo-curable PEGMA bioink into a chondral defect ${ }^{100}$. (B) Biofabrication of osteochondral tissue equivalents where microextrusion bioprinting was used to create a bi-layered construct containing chondrocytes in the cartilage layer and osteoprogenitors in the bone layer. Distinctive tissue formation was observed in the system in vivo as demonstrated by localised ALP staining in the bone region and collagen IV in the cartilage region ${ }^{81}$. (C) Bioprinting of 
complex shaped cartilaginous tissues using clinically compliant biomaterials. The bioink was composed of two FDA compliant polysaccharides (alginate, gellan) and combined with clinically available cartilage derived particles. (a-f) Using patient specific data, anatomically accurate, auricular shaped constructs containing overhanging layers could be printed with the ink. (g-j) The process could be leveraged to printed meniscal shaped constructs that could be manipulated by surgeons and sutured into a focal meniscal defect. (k-m) The system could also be used to print tissue templates in the geometry of the intervertebral disc and nose, further demonstrating the versatility of the approach ${ }^{91}$

\subsection{Bioprinting in Bone Tissue Engineering (BTE)}

Osteoarthritis is a disease that can affect both the articular cartilage and the underlying subchondral bone. Furthermore, traumatic joint defects often penetrate or progress into the underlying subchondral bone. As a result, there has been a significant amount of research devoted to developing implants for osteochondral defect repair. Many of the bioprinting technologies which have been described above for CTE have also been widely used for BTE. This section will describe the key developments in bioprinting and bioinks for BTE.

\subsubsection{Developing Bioinks for BTE}

One of the most common natural materials used for hydrogel based BTE constructs is alginate ${ }^{101,102}$. As discussed previously, alginate is commonly used as a bioink, making it a promising candidate for bioprinting implants for bone regeneration ${ }^{103,104}$. For example, when implanted in conjunction with BMP-2, bioprinted alginate constructs can lead to bone formation after 12 weeks in vivo ${ }^{104}$. However, both of these studies used a high molecular weight alginate which is easier to print with as it has a higher viscosity than other molecular weight alginates. This increase in molecular weight also significantly decreases its degradability, which is a key consideration for BTE. Both studies saw significant amount of 
alginate still present after 12 weeks in vivo. Moreover, there was no tissue formation or vessel infiltration within the gel itself, only in the surrounding area. Other studies using alginate based constructs for bone regeneration have reported similar problems ${ }^{105-108}$. This motivates the development of lower molecular weight alginate bioinks with faster degradation rates for BTE applications ${ }^{108}$.

In general, the leading limitation to the use of hydrogels alone for bone regeneration is their limited osteoinductivity and poor mechanical properties. This has motivated the development of composite bioinks with enhanced mechanical properties and osteo-inductivity. Wang et al. found that a bioink of both gelatin and alginate seeded with human derived adipose stem cells could induce bone matrix formation when subcutaneously implanted for 8 weeks in nude mice ${ }^{109}$. Another study created a methacrylated gelatin bioink with encapsulated bone marrow MSCs and collagen microfibers bound to BMP-2. When compared to a bioink encapsulated with bone marrow MSCs alone and cultured in osteogenic medium, the bioink with bone marrow MSCs and BMP-2 bound collagen microfibers induced faster osteogenesis of MSCs compared to those cultured in the presence of osteogenic growth factors after 14 days in vitro ${ }^{110}$. Campos et al. investigated a composite bioink of collagen and agarose, and found that by combining the thermos-responsive agarose hydrogels with collagen type 1 , the mechanical stiffness significantly improved compared to collagen type 1 alone ${ }^{111}$. Other studies have looked into adding bioglass particles to increase the mechanical stability of the hydrogel ${ }^{112,113}$. These studies have found that the addition of the bioglass can significantly improve the mechanical properties of the bioink ${ }^{113}$ whilst also producing a printable, porous material that can be used for generating BTE scaffolds ${ }^{112,113}$. Micro-carriers or small particles $(100-400 \mu \mathrm{m})$ of polymers such as PLA (poly(lactic) acid) or polyethylene have also been shown to increase the mechanical strength of a hydrogel ${ }^{114,115}$. Leveto et al. investigated the effect of adding micro-carriers to GelMA had on the mechanical properties of the hydrogel. 
The results showed not only were the mechanical properties significantly enhanced but the differentiation potential of human MSCs was also improved ${ }^{116}$. Wüst et al. investigated a combination of gelatin, alginate, and hydroxyapatite and found that the Young's modulus was significantly increased if $8 \%$ hydroxyapatite was added to the hydrogel ${ }^{117}$. However, even with a composite hydrogel the natural based hydrogels will still have a relatively low stiffness, in the $\mathrm{kPa}$ range, compared to bone which is in the GPa range.

Other investigators have explored the use of synthetic polymers within bioinks for BTE applications. Inkjet printing GelMA with added PEG significantly improved the resultant mechanical properties compared to GelMA constructs alone. Other studies investigated the addition of hydroxyapatite within a gelatin ${ }^{118}$ and polymer ${ }^{119}$ based bioink to increase the mechanical properties of the constructs. These studies found that the mechanical properties of such scaffolds increased to within the range of trabecular bone of the same density $(3.8-4$ $\mathrm{MPa}$ ). Although these hydrogels are within the range of cancellous bone, they are still lower than other rapid prototyping BTE strategies such as fused deposition modelling (FDM). PLGAPEG-PLGA ${ }^{120}$, PLGA alone ${ }^{121}$, PLA alone ${ }^{122}$, PCL alone ${ }^{123}$, and PCL-PLGA-TCP ${ }^{124}$ printed constructs have also been shown to have increased stiffness with moduli within the range of 57.4-244 MPa respectively, which is closer to the range for cortical bone ${ }^{125,126}$. This had led to increased interest in strategies where FDM can be integrated with microextrusion bioprinting to engineer composite reinforced tissues for BTE. This will be discussed in section 2.3.2 and

\subsection{3.}

\subsubsection{Bioprinting and Vascularisation in BTE}

Ensuring the successful vascularisation of TE constructs is arguably the most challenging hurdle to overcome in the field of BTE today ${ }^{127}$. As a result, there has been increased interest in using bioprinting technology to accelerate vascularisation of tissue 
engineered constructs for BTE. For example, a number of studies have explored whether the incorporation of microchannels into TE constructs can enhance vascularisation and subsequent bone formation in vivo ${ }^{53,128,103}$. For example, it has been demonstrated that the incorporation of microchannels into MSC seeded alginate hydrogels, achieved using bioprinting technology, could enhance vessel infiltration compared to a solid non-porous controls after 14 days in vivo ${ }^{103}$. Other studies, have used bioprinting to allow for spatial regulation of specific growth factors. Park et al. printed PCL fibers and within these fibers on the periphery co-printed dental pulp stem cells encapsulated in collagen type 1 with BMP-2 and in the centre printed a composite of gelatin and alginate with VEGF (Fig 2a). Microvessels were newly formed in the centre of the printed construct, and angiogenesis from the host tissue was also observed. Interestingly, vascularisation was enhanced by localising of VEGF presentation to central regions of the construct (Fig 2b). In another study, hierarchical vascularised bone biphasic constructs were bioprinted using a novel dual bioprinting approach ${ }^{129}$. Regional localisation of VEGF and BMP-2 was achieved using a novel thiol-ene click reaction which resulted in formation of a vascular network within the implant. In another approach, it was demonstrated that bioprinted scaffolds that facilitated the sustained long-term release of VEGF from a matrigel/alginate bioink could be used to enhance vascularisation in vivo ${ }^{130}$. 
A

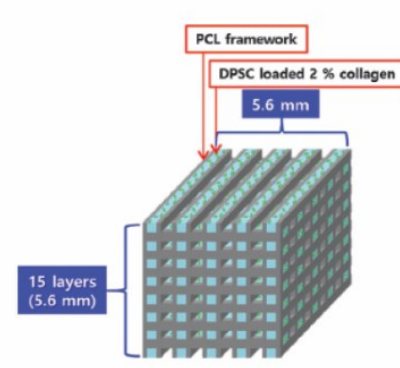

Group 1

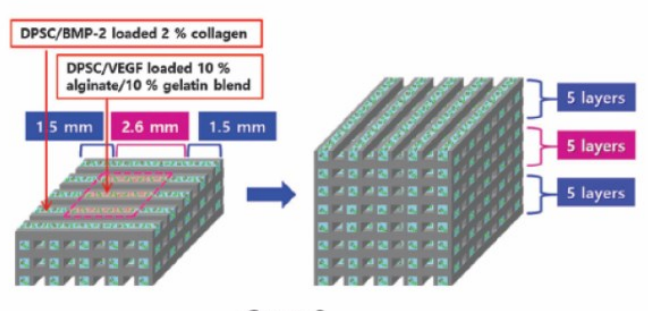

B
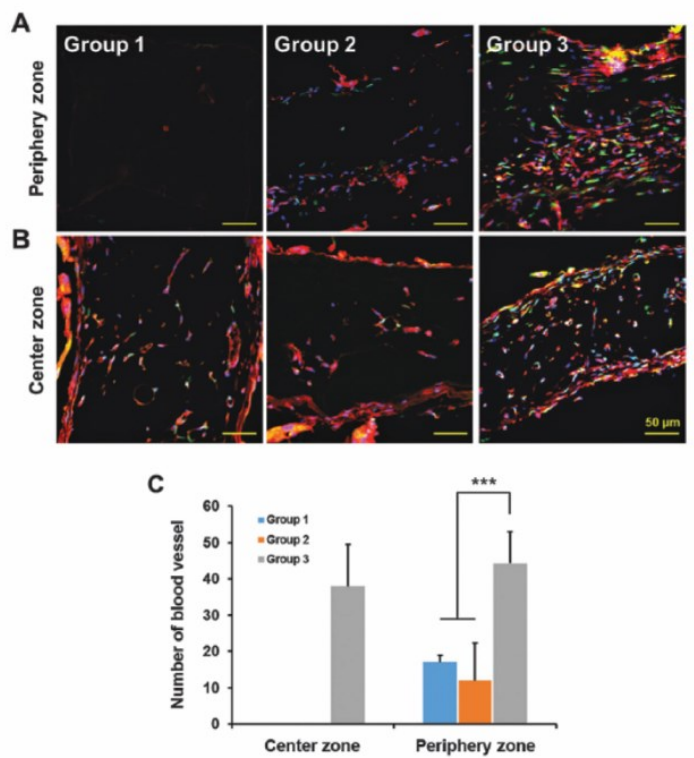

Figure 2: 3D printing technology to control BMP-2 and VEGF delivery spatially and temporally to promote large-volume bone regeneration ${ }^{131}$. (A) Outline of experimental groups. In group 3, region specific dual growth factor delivery was achieved by depositing the central portion of the scaffold with alginate/gelatin/VEGF and the outer portion of the scaffold was filled with collagen/BMP-2. This was compared to homogenous distributions of each growth factor (Group 1\&2). (B) Analysis of vessel formation at different sites in the implanted constructs after 28 days in vivo. (a-c) Higher levels of vessel formation were observed in both central and peripheral regions of group 3 where VEGF was localised to the central region of the construct. 


\subsection{Bioprinting of Composite Reinforced Tissues}

One of the major limitations with using hydrogel based bioinks is that they are often mechanically weak, and alone are not capable of supporting loading within a joint environment. To overcome this limitation, multi-material bioprinting approaches have been developed where a "soft" bioink can be reinforced with "stiffer" biocompatible and biodegradable polymer $32,33,51,83,99,132$. The most common approach is to co-extrude a stiff thermoplastic polymer such as PCL using FDM, alongside a bioink containing cells using microextrusion bioprinting 32,53,62. In this section we will first introduce fused deposition modelling. Next, this will be followed by a description of how the technology can be integrated with microextrusion bioprinting to engineer composite reinforced tissues for CTE and BTE.

\subsubsection{Fused Deposition Modelling (FDM)}

FDM is an additive manufacturing technology used for production, prototyping and modelling applications. As cells cannot be incorporated during the printing process due to high processing temperatures FDM is not technically considered bioprinting, however it is commonly used for producing porous scaffolds for TE ${ }^{133}$. FDM printers use a thermoplastic filament which is heated above its melting point and extruded onto a platform in a layer-bylayer process. The key advantage of FDM is that scaffolds with highly interconnected pore geometries and channels sizes can be fabricated rapidly. By varying process parameters such as the extrusion pressure, nozzle diameter and deposition speed it is possible to print scaffolds with a wide range of filament diameters and porosities ${ }^{134,135}$. Another key advantage of these scaffolds is that they are mechanically strong possessing mechanical properties in the range of articular cartilage and cancellous bone ${ }^{25,134-138}$. 
In one of the earliest applications of biofabrication technology in CTE, porous scaffolds were fabricated from a poly(ethylene glycol)-terephthalate poly(butylene terephthalate) (PEGT/PBT) block co-polymer using FDM ${ }^{139}$. Scaffolds with varying pore geometries were seeded with bovine articular chondrocytes and shown to support robust cell proliferation and matrix synthesis. The same group also compared scaffolds generated with FDM to more traditional particulate leached scaffolds ${ }^{140}$. Cell viability in central regions of the scaffold produced via FDM was significantly higher than the controls, with the authors demonstrating this was due to superior nutrient and oxygen diffusion in the orientated pores of the FDM scaffold. In another early approach, a biphasic osteochondral scaffold composed of a PCL-TCP phase for the bone region, and a PCL-fibrin phase for the cartilage phase was generated using FDM ${ }^{141}$. The scaffolds were seeded with bone marrow derived MSCs and implanted into osteochondral defects in rabbits. Post-implantation, $\mu \mathrm{CT}$ analysis revealed significant regeneration in the bone phase, however, cartilage repair was limited throughout. Porous PEOT/PBT scaffolds produced using FDM and seeded with MSCs have also been explored for osteochondral defect repair ${ }^{142}$. Compared to empty controls, no significant improvement in repair was observed highlighting the challenges faced in regenerating cartilage defects. Recently, more sophisticated approaches have been developed in an attempt to develop spatially graded cartilage tissues, with architectures better mimicking the native tissue. For example, gradients in pore size, pore geometry, surface energy and stiffness can be tuned using FDM resulting in improved MSC chondrogenesis in vitro ${ }^{143-145}$. It is yet to be seen whether these improvements in vitro will translate into enhanced cartilage defect repair in vivo. 
3D printing of synthetic polymers also represents a promising material for bone tissue repair as they provide the necessary mechanical strength, are easy to fabricate, are cost effective, biocompatible and have tuneable degradation rates. The most common synthetic polymer used for BTE scaffolds is PCL ${ }^{146,147}$ and PLGA ${ }^{121,148}$, with both shown to support bone regeneration ${ }^{149}$. However, although the synthetic polymers generate mechanically stable construct they do not contain the osteoconductive compounds such as tri-calcium phosphates (TCP) and hydroxyapatite (HA). Taking this into account, more recent studies have begun to investigate the potential of 3D printed composite structures with filaments consisting of both ceramics and synthetic polymers ${ }^{150-152}$. Heo et al. investigated the regeneration potential of nHA/PCL composite scaffold within a rabbit tibial segmental defect model. After 8 weeks, dense bone tissue formation was observed throughout all of the constructs ${ }^{150}$. However, although dense bone had formed in and around the scaffold a large proportion of the scaffold was still present after 8 weeks in vivo. Kim et al. investigated the regeneration potential of TCP coated PLGA constructs within a rabbit femoral defect model. At 12 weeks, the TCP coated constructs showed a trend towards increased bone formation however, new bone formation was $<10 \%$ across the treatment groups ${ }^{151}$. Finally, Reichert et al. investigated if $3 \mathrm{D}$ printed constructs that display similar mechanical properties to cancellous bone might be suitable to augment segmental ovine bone defects of the tibia. PCL-TCP and poly(L-lactide-co-D,Llactide) (PLDLLA)-TCP-PCL scaffolds were implanted in within an ovine segmental defect for 12 weeks. After 12 weeks only minor external callus and bone formation was observed in the scaffold groups, with the least amount of bone formation present within the PCL-TCP composite constructs ${ }^{152}$. In general, the results from these studies show the potential for the use of 3D printed composite constructs for the repair of large segmental defects, however significant room for improvement is noted in many of these studies. 


\subsubsection{Bioprinting of Reinforced Constructs for Cartilage and Osteochondral Tissue Engineering}

It has been shown that PCL and cell containing bioinks can be co-printed in a layer-bylayer fashion to build 3D constructs for CTE (Fig 3a) ${ }^{153}$. Even though the PCL component is heated to approximately $60^{\circ} \mathrm{C}$ to facilitate extrusion, cells in the bioink phase remain viable post-printing ${ }^{83}$. The mechanical properties are significantly improved with the incorporation of PCL filaments and by modulating the percentage of reinforcing polymer, constructs with compressive equilibrium moduli in the range of articular cartilage can be achieved ${ }^{83}$. A subcutaneous analysis of a PCL reinforced alginate construct embedded with chondrocytes and supplemented with chondrogenic growth factor TGF- $\beta 3$ reported collagen type II and sGAG deposition ${ }^{51}$. In a similar approach, bi-layered constructs with spatially distinct regions of ECM materials and growth factors were created for osteochondral tissue engineering ${ }^{154}$. Human MSCs were combined with atelocollagen and BMP-2 in the bone region, and hyaluronic acid and TGF- $\beta 3$ in the cartilage region. The construct was reinforced using a PCL frame and demonstrated heterogeneous tissue development in an osteochondral defect model. With such approaches there is little or no mechanical interaction between the reinforcing component and the bioink network. This can be a problem, particularly at higher strains, which can lead to mechanical disintegration of the implant upon the application of mechanical loads. Recently it has been demonstrated that by covalently crosslinking the hydrogel and thermoplastic phases together it is possible to increase the interface strength ${ }^{132}$. This resulted in improved resistance to repetitive rotational and axial loading. It should be noted here that co-printing approaches can influence the mechanical properties of the final construct. For example, layer-by-layer bioink deposition can interfere with the adhesion of PCL fibers during co-printing which can significantly reduce the resultant mechanical properties of the engineered construct ${ }^{155}$. 
The degradation rate of PCL, which can be up to 2 years ${ }^{156,157}$, is a potential limitation with such multi-material approaches, as residual filaments can act as a barrier to tissue formation. Recently alternative polymers based on PCL have been developed to overcome these potential limitations. A poly (hydroxymethylglycolide-co-caprolactone) (PHMGCL) polyester which has a greater hydrophilicity than unmodified PCL due to the addition of hydroxyl groups has been developed which loses $\sim 60 \%$ of its initial weight after 3 months in vivo ${ }^{147,158}$. An extensive review on the use of PCL for additive biomanufacturing and other biomedical applications is available elsewhere ${ }^{157}$. An alternative approach is to use a faster degrading polymer such as PLGA. PLGA co-polymers composed of PLA and Poly(glycolic acid) (PGA) can be developed which degrade more rapidly due to the hydrophilic nature of PGA which facilitates hydrolysis of the polymer backbone ${ }^{159}$. The rate of degradation can be controlled by varying the ratio of lactide and glycolide in the copolymer with higher percentages of glycolide resulting in accelerated degradation rates ${ }^{159}$. One drawback of using PLGA is that the build-up of acidic by-products that occur following its degradation can cause adverse inflammatory responses ${ }^{160,161}$.

Osteochondral constructs have also been fabricated using a co-printing approach with PLGA rather than PCL ${ }^{162}$. The chondral layer was formed using an alginate bioink combined with cartilage derived ECM and an alginate/hydroxyapatite bioink was used for the osseous layer. Another way to overcome limitations with residual PCL material is to reduce the amount of the reinforcing polymer used by increasing the porosity of the reinforcing phase. This has led to an increased interest in the use of melt-electrowriting (MEW) which is an emerging technology that combines key aspects of melt-electrospinning and FDM. The process is similar to FDM except that a voltage is applied between the nozzle tip and the printing platform to draw filaments of material from the nozzle tip onto the platform. By carefully controlling the relative motion between the printhead and the collector it is possible to print the fibers with a 
high degree of accuracy. Highly organised networks of fibers with diameters down to $0.8 \mu \mathrm{m}$ have been printed with this technology ${ }^{163-165}$. This is a major advantage over traditional FDM printing where it is difficult to print fibers with diameters lower than $100 \mu \mathrm{m}$.

PCL fibers produced using FDM are usually large $(>150 \mu \mathrm{m}$ in diameter) and the porosity of the resultant scaffolds are usually less than $80 \%$. To overcome this, GelMA hydrogels were reinforced with highly porous PCL scaffolds (porosity 98\%-93\%, fiber diameter 19-50 $\mu \mathrm{m}$ ) produced using MEW rather than FDM ${ }^{33}$. The stiffness of the resultant composites were within the native cartilage range and importantly the yielding strains $(\sim 25 \%$ $40 \%$ strain) of the composites were much higher than equivalent scaffolds produced using FDM $(\sim 8 \%)$. This is an important consideration for CTE as strains of greater than $10 \%$ are repeatedly experienced during locomotion ${ }^{166}$. In addition, cyclic mechanical tests demonstrated that the scaffold could recover after 20 cycles at $20 \%$ strain. Another hybrid strategy incorporated inkjet printing with electrospinning ${ }^{98}$. A PCL electrospun mat was alternated with a fibrin-collagen solution containing chondrocytes for 5 layers until a thickness of $1 \mathrm{~mm}$ was reached (Fig $3 \mathrm{~b}$ ). The fabricated constructs formed cartilage matrix both in-vitro and in-vivo as evidenced by the deposition of type II collagen and sGAG. Recently, composite soft cartilage constructs were fabricated by combining PCL microfibers produced using MEW and a highly negatively charged star-shaped poly(ethylene glycol)/heparin hydrogel (sPEG/Hep) ${ }^{167}$ (Fig 3c). The hydrogel network mimicked the function of the cartilage proteoglycan network and the PCL fibers mimicked the function of the cartilage collagen fiber network. The resultant constructs exhibited mechanical anisotropic, nonlinear and viscoelastic behaviour analogous to native cartilage. 
A

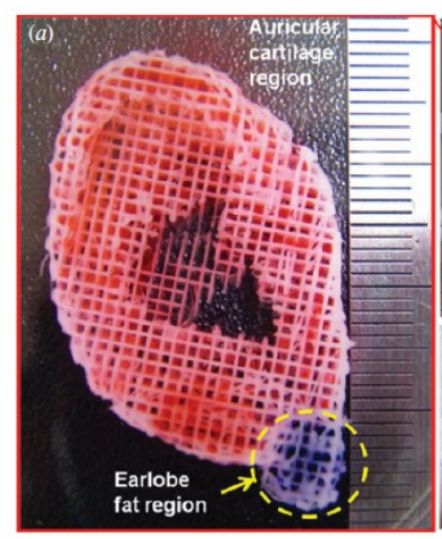

B
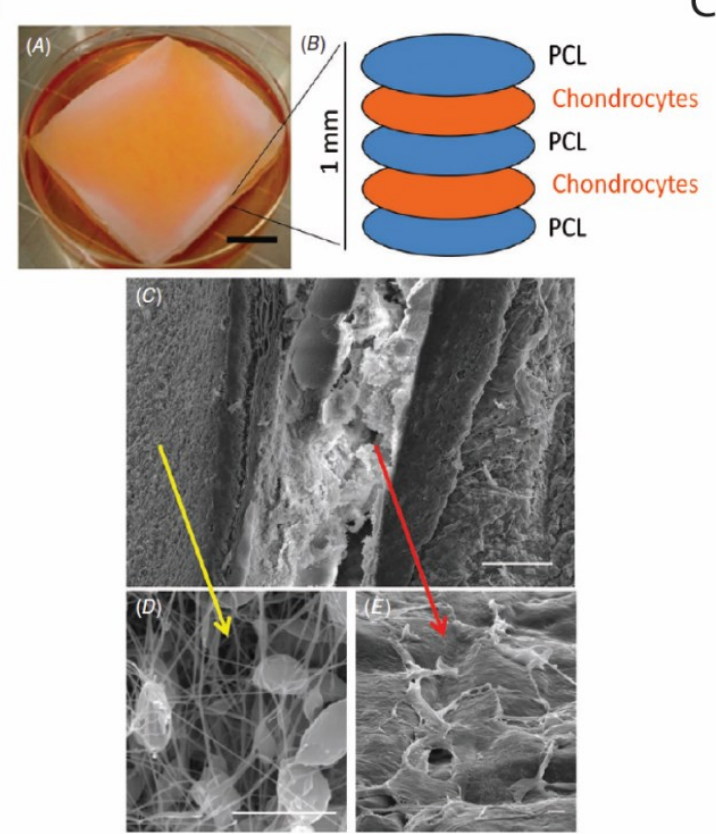

C
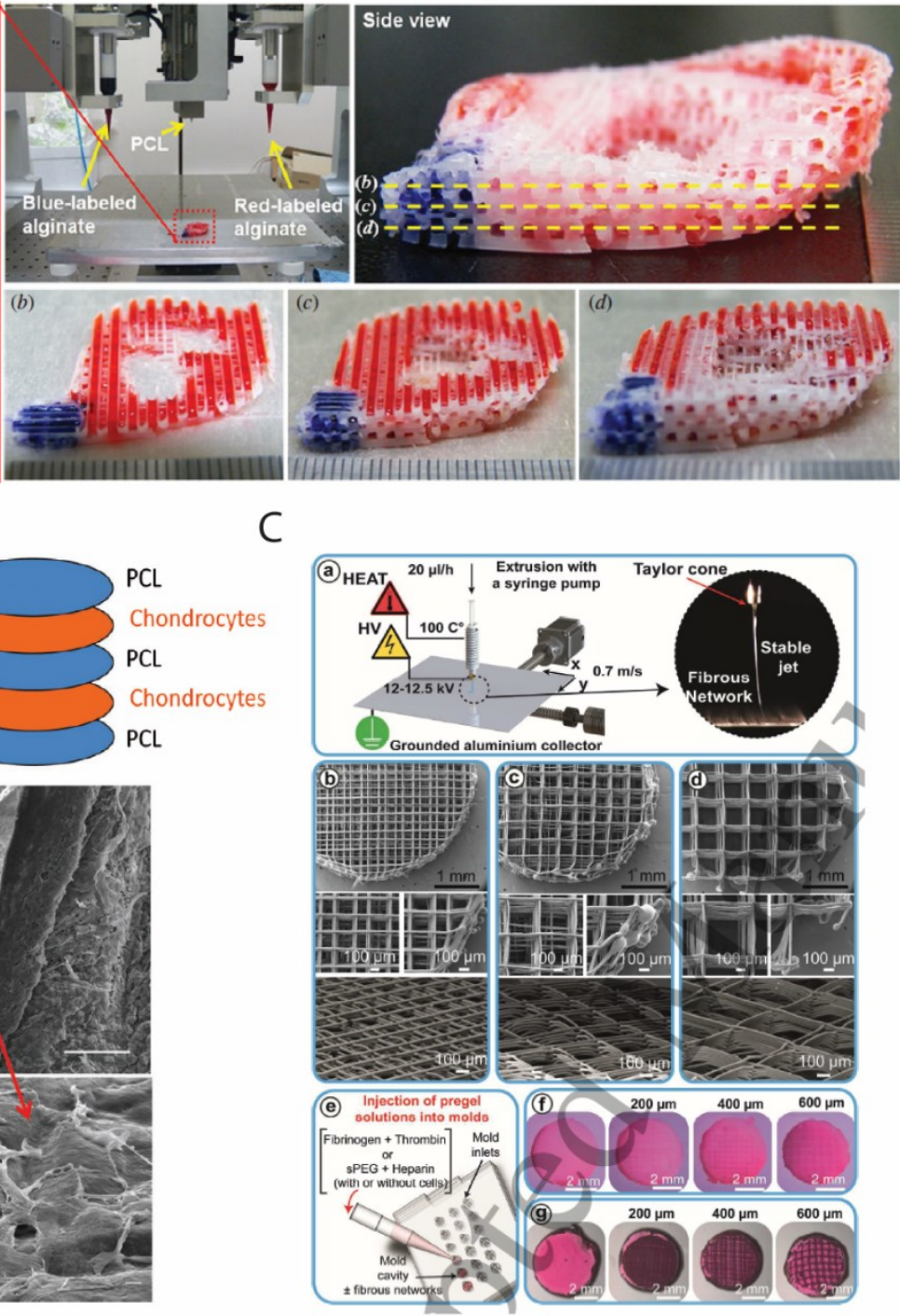

Figure 3: Biofabrication of Composite Constructs for CTE (A) Dual bioprinting of composite, anatomically accurate, PCL/bioink constructs for auricular CTE ${ }^{153}$. (B) Hybrid printing of mechanically improved constructs for CTE. The constructs were produced by inkjet bioprinting rabbit chondrocytes in a fibrin collagen bioink onto electrospun PCL microfibers in a layer-by-layer fashion. (C) (a) Biofabrication of soft network reinforced constructs for CTE. Graphical representation of MEW device and operating parameters, (b-d) SEM of crosshatch structure produced with $200 \mu \mathrm{m}, 400 \mu \mathrm{m}$ 
and $600 \mu \mathrm{m}$ fiber spacing. Preparation of composite constructs following injection of highly negatively charged sPEG/Hep hydrogels ${ }^{167}$

\subsubsection{Bioprinting of Reinforced Constructs for BTE}

Co-printing approaches have also been explored for BTE ${ }^{53,128}$. In one approach, a cell laden bioink containing adipose derived stem cells was co-deposited alongside a mixture of PCL and tricalcium phosphate (TCP) ${ }^{128}$. The constructs were implanted in cranial defects in rats and demonstrated good integration and evidence of bone repair. In a similar approach, vertebrae shaped constructs were co-printed using PCL and alginate bioink containing MSCs 53. The constructs were chondrogenically primed in vitro and once implanted in vivo, were capable of forming vascularised bone organs via endochondral ossification.

\subsection{Whole Joint Resurfacing}

The majority of TE studies to date have focused on treating focal cartilage and osteochondral defects. However diseases such as OA effect the entire joint surface. A number of studies have explored using FDM to engineer templates for biological joint resurfacing. Ding et al (2013) employed a modular approach to engineer tissue specific, biphasic scaffolds for regeneration of the femoral head. First a porous interconnected PCL/Hydroxyapatite scaffold was fabricated using FDM for the osseous phase and combined with PLGA scaffold for the chondral layer ${ }^{168}$. Autologous chondrocytes and BMSCs were seeded in the chondral and osseous regions respectively with the construct demonstrating histological evidence of specific cartilage and bone matrix formation along with a well-integrated osteochondral interface following subcutaneous implantation.

CAD/CAM technology has also been used to tissue engineer constructs potentially suitable for whole joint regeneration. Anatomically accurate constructs were generated from 
$\mu$-CT scans to form mechanically functional scaffolds with a porous internal architecture using FDM ${ }^{169}$. Rabbit chondrocytes were seeded onto the scaffolds and cultured in chondrogenic media for 21 days prior to implantation (Fig 4a). Tibial and femoral osteotomies were created removing the articular surfaces within the knee and the tissue engineered replacements were fixed within the joint. Histological analysis of the joint post-implantation demonstrated restoration of two congruent articulating surfaces with evidence of bone tissue formation and host integration. However the repair tissue was mainly fibrous-like throughout, with little evidence of hyaline cartilage development, highlighting the importance of providing the appropriate biological cues in such large defect models. In an important study by Lee et al. (2010) the surface morphology of a rabbit proximal humeral joint was captured using laser scanning and reconstructed by FDM to engineer an anatomically accurate graft using PCL/Hydroxyapatite ${ }^{170}$. The scaffold was infused with TGF- $\beta 3$ and implanted into the unilateral proximal humeral condyles of skeletally mature rabbits. Remarkably all animals that received TGF- $\beta 3$ infused scaffolds resumed weight bearing and locomotion 3-4 weeks after surgery (Fig 4b). Hyaline-like cartilage was found to cover the entire surface of the infused scaffolds, with regeneration of a well vascularised subchondral bone region. Importantly compressive and shear moduli of the regenerated cartilage were comparable to native articular cartilage. This study demonstrated that the entire articular surface could be regenerated through homing of endogenous cells into a porous interconnected scaffold. The spatial incorporation of bioactive factors within $3 \mathrm{D}$ printed constructs is a promising strategy for developing gradients of biological signals. The localised presentation of these signals can make it possible to engineer tissues that better recapitulate the complex architecture of their native counterparts. Recently, this approach has been explored through the embedding of growth factor loaded PLGA microspheres into PCL microfibers for controlled release, resulting in enhanced regeneration of temporomandibular joint defects ${ }^{171}$. 
A
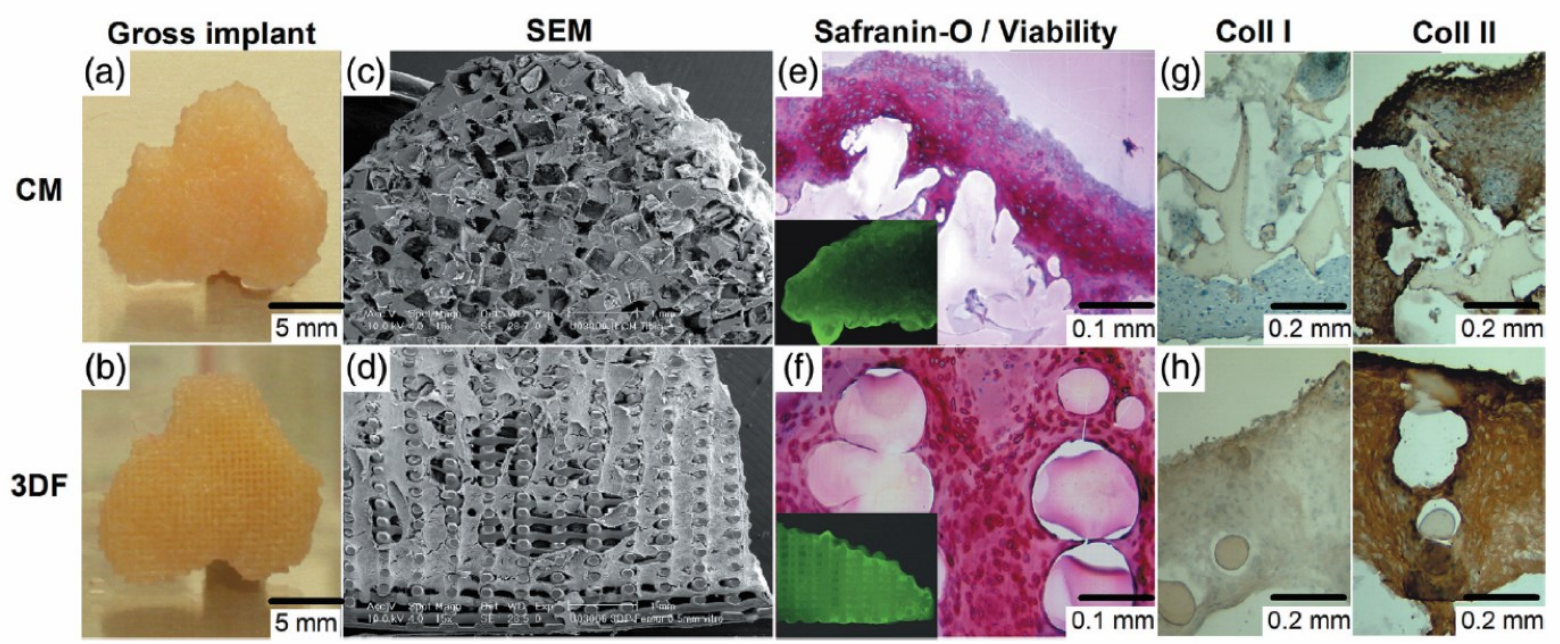

B

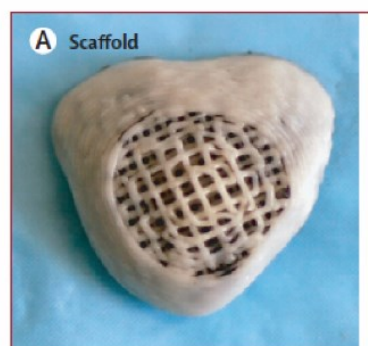

E

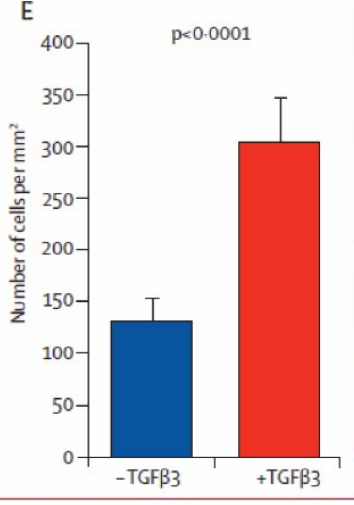

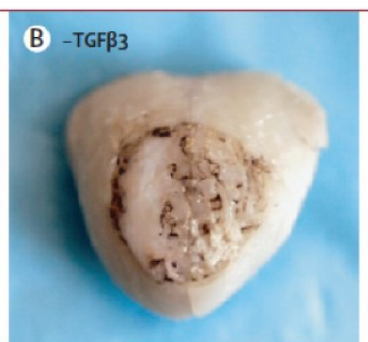

F - TGF 3
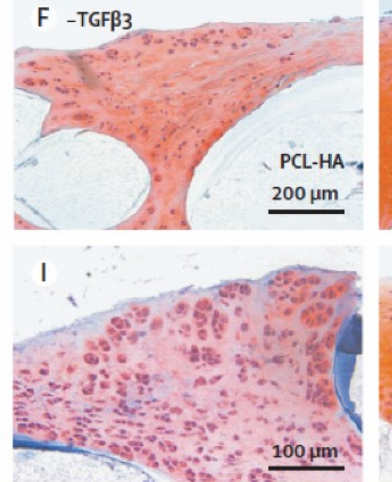
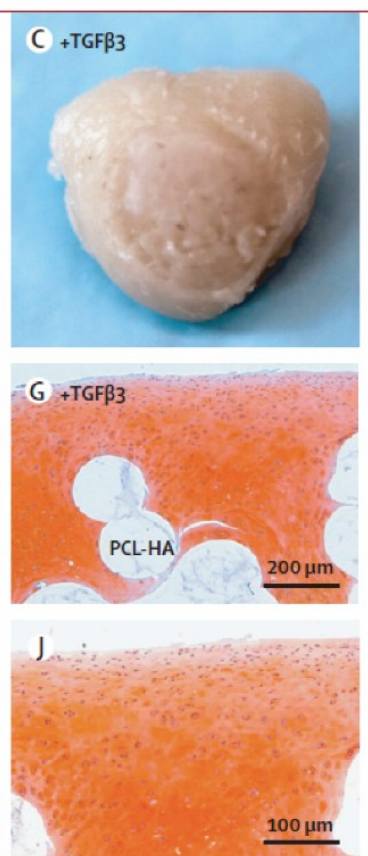

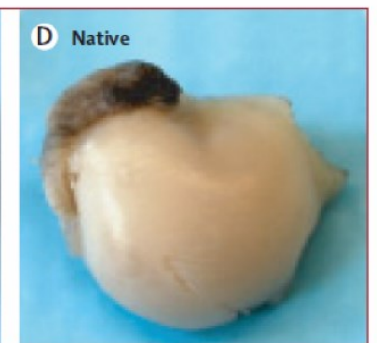

$\mathrm{H}$
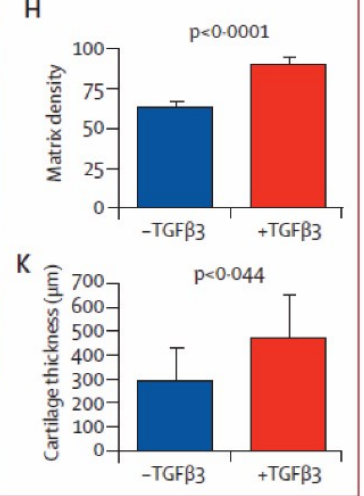

Figure 4: Biofabrication as a tool for joint resurfacing. (A) Tissue engineered cartilage templates for joint replacement were produced by seeding chondrocytes onto tibial shaped scaffolds, generated using FDM and particulate leaching ${ }^{172}$. Pre-implantation, (c, d) SEM analysis, (e, f) Safranin-O/live-dead analysis and (g, h) immunohistochemical staining for collagen I, II demonstrated robust chondrogenesis within both systems. However, compared to particulate leaching, the scaffolds produced using FDM supported superior cell viability and matrix production in central regions. This is a key 
consideration when attempting to engineer scaled up implants which will be required for joint resurfacing strategies. (B) Regeneration of the articular surface of a rabbit synovial joint using a cell homing approach where bioscaffolds produced using FDM were implanted into the shoulder joint of New Zealand white rabbits following a shoulder joint osteotomy. (a-d) Post-implantation, macroscopic imaging demonstrated the addition of TGF- $\beta 3$ improved cartilage regeneration compared to empty scaffold only controls. (e-k) Quantification of the matrix density, cartilage thickness and number of cells $/ \mathbf{m m}^{2}$ following histological staining further demonstrated superior restoration of the articular surface in the joint with the addition of TGF- $\beta 3$. 


\section{Future Directions:}

\subsection{Cartilage Tissue Engineering}

It is clear that bioprinting technology holds tremendous promise for CTE. The ability to pattern heterogeneous constructs makes it possible to engineer complexities otherwise unattainable with subtractive manufacturing techniques. A number of novel bioinks have been developed that push the boundaries of the traditional biofabrication window. These inks are highly printable, but also biologically relevant, and can support cell proliferation, differentiation and tissue production ${ }^{87,91}$. However, a number of key challenges must be addressed before bioprinted implants become widely available for joint repair.

\subsubsection{Biofabrication of Mechanically Functional Cartilage Tissues}

Since bioinks are often hydrogel based they are generally mechanically weak and not suitable for deployment in load bearing locations. While significant mechanical reinforcement can be achieved by co-printing thermoplastic polymers ${ }^{83}$, it is not clear whether such implants will be sufficiently tough to withstand long term repetitive loading in a joint environment. For example, scaffolds produced using FDM typically permanently deform at strains levels above $8-10 \%{ }^{33}$. In addition, it has been demonstrated that polymer reinforced hydrogels disintegrate at the boundary between the two phases under physiological loads ${ }^{132}$. This problem can be at least partially addressed by improving the interface binding through chemical modification of the materials ${ }^{132}$. However, it is still unclear whether these modifications will be sufficient to withstand repetitive long term loading in a joint environment. Typically the mechanical properties of tissue engineered cartilage are only measured in compression, and other potential failure modes like shear, are usually ignored. High levels of shear, tension and compression will be experienced in the joint and implants that can withstand repetitive combinations of these loading patterns will be required. Bioreactors models of the joint, that can mimic joint loading 
patterns, should be used as a checkpoint before attempting to progress into large animal models 132,173

Another promising approach to developing mechanically functional implants for joint resurfacing could involve the development of tough bioinks based on interpenetrating network (IPN) hydrogels. IPNs are a class of materials formed by combining multiple polymer networks. By combining a suitably contrasting primary and secondary network that can support IPN entanglement and energy dissipation, it is possible to engineer extremely tough and flexible hydrogels with mechanical properties comparable to high load bearing tissues ${ }^{174,175}$. Recently, a double network bioink, formed by combining PEG and alginate, was combined with a nano-silicate clay to create a mechanically tough ink with mechanical properties in the range of native articular cartilage ${ }^{176}$. These approaches will likely find utility in the future of cartilage tissue bioprinting. The challenges will arise in balancing the mechanical and biological functionality of the implant as IPN networks are typically dense making it difficult for embedded cell populations to produce de novo matrix components.

\subsubsection{Bioprinting of Stratified Cartilage Tissues and Osteochondral Tissue Interfaces}

As mentioned previously, articular cartilage is a highly anisotropic tissue and its composition and organization varies greatly with depth. Surprisingly, few studies have explored the possibility of bioprinting gradients of biological factors in order to recreate the zonal properties of articular cartilage and its interface with the underlying bone. Now that a wide range of suitable bioinks are available, future work should explore how combinations of biological cues can be used to engineer biomimetic tissue gradients for CTE. During tissue development, repair and homeostasis cells experience and respond to gradients of chemical and physical cues. These gradients can influence a number of different cellular behaviours including proliferation, migration and differentiation. In a tissue engineering context, physical 
gradients such as pore size and substrate stiffness, or biochemical gradients such as growth factor presentation, can be introduced into a construct. It has been demonstrated that biochemical gradients, such as the graded presentation of growth factors, can lead to the development of heterogeneous engineered tissues. For example, an overlapping graded presentation of recombinant human bone morphogenic protein 2 (rhBMP-2) and insulin-like growth factor (rhIGF-I) has been shown to direct localised osteogenic and chondrogenic differentiation of MSCs in silk scaffolds ${ }^{177}$. In addition, it has been demonstrated that gradients of TGF- $\beta$ in MSC seeded hydrogels, which result from a combination of high-affinity binding interactions and a high cellular internalisation rate, can lead to the development of highly heterogeneous cartilage tissues ${ }^{178}$.

These studies all highlight the benefits of incorporating biochemical and physical gradients into cartilage and osteochondral tissue engineering strategies. Surprisingly, given the additive layer-by-layer nature of biofabrication technology, relatively few studies have explored the possibility of developing gradients using bioprinting approaches. In an early approach, overlapping gradients of IGF-II and BMP-2 have been deposited onto a fibrin substrate and shown to spatially direct the fate of $\mathrm{C} 2 \mathrm{C} 12$ cells toward an osteogenic lineage 179. Future work should look to expand on these approaches to direct the development of both stratified cartilage tissues and the osteochondral interface. For example, combinations of physical and biochemical gradients could be used to engineer more native like cartilage tissues. As an example of this, a novel dual syringe system was developed to produce a combination of physical and chemical gradients, including substrate stiffness, RGD ligand presentation and growth factor concentration, to control differentiation of MSCs along chondrogenic and osteogenic lineages ${ }^{180}$. In another multifactorial approach, it was demonstrated how combinations of growth factors (BMP-7, IGF-1 and hydroxyapatite), substrate stiffness values (80 $\mathrm{KPa}, 2.1 \mathrm{MPa}$ and $320 \mathrm{MPa}$ ) and nanofiber alignments (horizontal, random, and 
perpendicular to the gel surface) could be used to direct the zone specific chondrogenic differentiation of MSCs ${ }^{181}$. Bioprinting technology could be used to further expand on these ideas to engineer cartilage tissues with a native like organisation. Future advances in bioprinting technology will likely make it possible to present multiple gradients of physical and chemical cues, across multiple length scales. For example, in a recent ground breaking study, a microfluidic bioprinting system capable of simultaneous spatial deposition of 7 distinct bioinks was developed ${ }^{182}$. The system was able to generate complex gradient structures highly suitable for tissue engineering applications.

\subsubsection{Towards Biofabrication of Anatomically Accurate Cartilage Tissues}

The layer-by-layer nature of bioprinting technology makes it easy to create anatomically accurate constructs for tissue engineering applications ${ }^{183}$. Imaging techniques such as magnetic resonance imaging (MRI) ad computed tomography (CT), that are widely used in the clinic, can be easily converted into formats compatible with biofabrication technology. Patient specific tissue engineered implants could therefore become a reality for sufferers of joints diseases such as OA. However, a number of challenges exist with current bioprinting software that must be addressed. Traditional biofabrication technology creates 3D constructs by continually adding $2 \mathrm{D} x-y$ patterns into the $\mathrm{z}$ plane. As a result, fibers are orientated parallel to printing platform and the outer surfaces of curved constructs are jagged and discontinuous in nature. This can make it challenging to create constructs with smooth outer surfaces. This is a key consideration for CTE as the main function of the tissue is to support smooth, pain-free articulation. Future work should explore strategies to orientate printed fibers in anatomically relevant orientations. Such capabilities could also lead to more biomimetic mechanical behaviour of resultant tissue engineered implants. 


\subsection{Bioprinting of Vascularised BTE Constructs}

As mentioned previously, achieving vascularisation is a major challenge for BTE today 127. The formation of blood vessels involves a complex set of cell-cell interactions. Endothelial cells must proliferate, elongate, undergo lumenogenesis and tubulogenesis and finally become stabilised through the association of pericytes. This process is relatively slow, the average growth rate of newly developing microvessels is only $\sim 5 \mu \mathrm{m} / \mathrm{h}{ }^{184}$, and it also produces networks which are heterogeneous and unpredictable. To improve the efficiency of vascularisation in TE tissues, many researchers are now attempting to prevascularize TE constructs to enable rapid anastomosis with host vasculature upon implantation in vivo. Multiple methods have been carried out including establishing microvessel networks within constructs through the self-assembly of endothelial cells in co-culture with supporting cells ${ }^{185-}$ 187 or electrochemical approaches ${ }^{188}$. Although these approaches can result in a more rapid establishment of a vascular network, the resulting networks are heterogeneous and unpredictable. 3D bioprinting technologies have shown great promise in recent years to overcome these issues by enabling microchannels to be directly fabricated within $\mathrm{TE}$ constructs. This enables higher control over the architecture of these vascular networks as well as providing the possibility to design locations for either natural or surgical vascular anastomosis.

One method to pattern perfusable vascular networks is to print sacrificial 3D filament networks within cell permissive hydrogels to create an interconnected vascular template within the construct ${ }^{189}$ Cytocompatible sacrificial materials such as gelatin ${ }^{190} 191$ carbohydrate glass, 189 agarose ${ }^{192}$ and fugitive inks such as pluronic F127193 194 can be easily printed within constructs and later removed to create an embedded hollow network of channels. These channels can be later lined with endothelial cells to create primitive vasculature. ${ }^{189,194}$ Recent 
advances in direct seeding techniques have shown that vessels as small as $20 \mu \mathrm{m}$ in diameter can be seeded with endothelial cells ${ }^{195}$.

Another more direct approach to achieve controlled vascular networks which is emerging in the field is to directly pattern cells without the need for a subsequent seeding step. Hammer et al. (2014) ${ }^{196}$ used a coaxial flow of alginate and calcium chloride solution to fabricate microfibers with sizes ranging 150-200 $\mathrm{mm}$. Cell laden microfibers were encapsulated within 3D hydrogels and then exposed the EDTA to dissolve the alginate microfibers to leave behind microchannels containing cells along the luminal surfaces. In a similar approach, perfusable vascular constructs were created using a direct 3D bioprinting approach ${ }^{68}$. A specially designed bioink consisting of GelMA, alginate and 4-arm poly (ethylene glycol)-tetra-acrylate (PEGTA) was used in combination with a multi-layered coaxial extrusion system. This made it possible to directly pattern hollow tubes of various diameters containing HUVECs in the lumen of the constructs.

Despite significant progress in bioprinting of vascular networks a number of challenges must still be addressed. Strategies that can direct and connect vascular networks across multiple length scales will be required when attempting to engineer larger bone tissues. The possibility of generating multiscale vascular networks using 3D bioprinting technology has been demonstrated ${ }^{197}$. Briefly, two larger vessels ( $\sim 1 \mathrm{~mm}$ lumen diameter) were printed in parallel and surrounded with a HUVEC/fibrin mixture. The larger vessels were next perfused, and over time microvessels self-assembled and integrated with the parent vessels to form a multi scale vascular network.

To date, most bioprinting approaches to produce prevascularised constructs involve relatively simple cell combinations. Recent advances in 3D bioprinting techniques are enabling more complex combinations of cell types and biomaterials to be co-printed. This can facilitate 
the fabrication of multicellular heterogeneous constructs. For example, Colosi et al (2016) developed a coaxial needle extrusion system capable of printing two bioinks simultaneously allowing for the deposition of heterogeneous fibers. ${ }^{198}$ They demonstrated the ability of this type of approach to produce a 3D HUVEC scaffold capable of supporting beating primary cardiomyocytes. In the future, similar approaches may contribute to the fabrication of rapidly stabilised microvessels through the parallel co-printing of endothelial cells with pericyte-like supporting cells.

The utility of presenting gradients of growth factors has also been demonstrated in the context of angiogenesis. In one example, endothelial cell migration was studied in collagen scaffolds with and without the presence of a VEGF gradient. In the graded presentation ECs migrated and formed sprouted structures, whereas in the controls significantly less sprouting and migration occurred ${ }^{199,200}$. In the context of TE, neovascularisation within constructs could be further promoted through the incorporation of angiogenic growth factor gradients, either by the direct immobilisation of growth factor gradients onto a scaffold 201,202 or by the inclusion of growth factor containing microspheres within a construct. ${ }^{177}$ In combination with bioprinting and biomaterial strategies, which would enable both spatial and temporal control over the delivery of growth factors, future approaches could utilise growth factor gradients to direct the vascularisation process.

Engineering the optimal biosignalling, scaffold architecture and cell combination for supporting both angiogenesis and osteogenesis in BTE constructs has yet to be realised. The aforementioned examples demonstrate that bioprinting is a highly adaptable process with great potential to play a significant role in the future of this research. 


\subsection{Bioprinting of Gene Activated Bioinks}

The incorporation of bioactive factors within bioprinted implants is a promising strategy for enhanced tissue regeneration. 3D bioprinted constructs can be used as a means to control the release of growth factors to promote lineage-specific differentiation of stem cells, vascularisation from the surrounding tissue, and enhanced healing. However, localising the presentation of signalling molecules can be challenging as hydrogels have diffusive transport characteristics. A potential way to overcome this limitation could be through nucleic acid delivery, via genes encoding for key signalling factors. Nucleic acid delivery allows for a physiological and sustained strategy in which the cell-mediated expression of the transgene guarantees authentic post-translational modifications and increased biological activity ${ }^{203}$. The localised presentation of nucleic acid acids encoding for genes associated with tissue growth and development may be a promising way to engineer complex tissues and interfaces. Additionally, compared to direct growth factor delivery, this strategy allows for a simpler way of simultaneous and sequential delivery of growth and transcription factors that could enhance the multifactorial processes of tissue formation ${ }^{204,205}$.

There are various ways to combine nucleic acid delivery with biofabrication techniques such as 3D printing (Fig 5). The nucleic acid of interest and its delivery mechanism (chemical, physical or viral) could be incorporated using a one-step approach during the biofabrication process by either its encapsulation into a printable biomaterial ${ }^{204,206,207}$, producing a gene activated bioink (GAB) (Fig 5c), or by the physical introduction of the genetic material into the required cell population due to the forces applied during the printing mechanism ${ }^{208,209}$ (Fig 5b). Porous bioprinted alginate constructs incorporating plasmid DNA (pDNA) encoding for the osteogenic growth factor BMP2 and calcium phosphate particles were able to efficiently transfect encapsulated MSCs over 14 days and promote their differentiation towards the 
osteogenic lineage ${ }^{206}$. These gene-activated constructs were also assessed in a goat iliac crest model showing enhanced bone repair ${ }^{210}$. More recently, nanohydroxyapatite-mediated gene delivery in a MSCs laden alginate hydrogel showed to be effective to direct MSC phenotype towards an endochondral or chondrogenic phenotype depending on the delivered genes ${ }^{204}$, confirming the potential of this approach for bioprinting of cartilage and osteochondral tissue interfaces. Inkjet printing was also used for the transfection of primary cells through the transient pores created in the cell membrane during the printing process ${ }^{209}$, achieving a transfection efficiency over $10 \%$ and with minimal detrimental effects over cell viability ${ }^{208}$. This promising approach might also allow for a fine spatial control over different transfected cell populations able to reproduce the bio-distribution of growth and transcription factors in native tissues.

In addition, delivery of nucleic acids such as RNA and DNA can be also incorporated into the scaffold after the biofabrication process in a two-step process (Fig 5) through biomaterial-based chemical interactions or direct loading onto the construct, facilitating its cellular uptake. Lentiviral vectors chemically immobilised in 3D orthogonally PCL scaffolds were able to transfect MSCs and drive cell-mediated transforming growth factor beta 3 (TGF$\beta 3)$ production in a sustained manner over 4 weeks of in vitro culture ${ }^{211}$; the over-expression of the growth factor increased the production of glycosaminoglycans (GAGs) and the expression of the chondrogenic markers aggrecan and collagen type II ${ }^{211}$. 

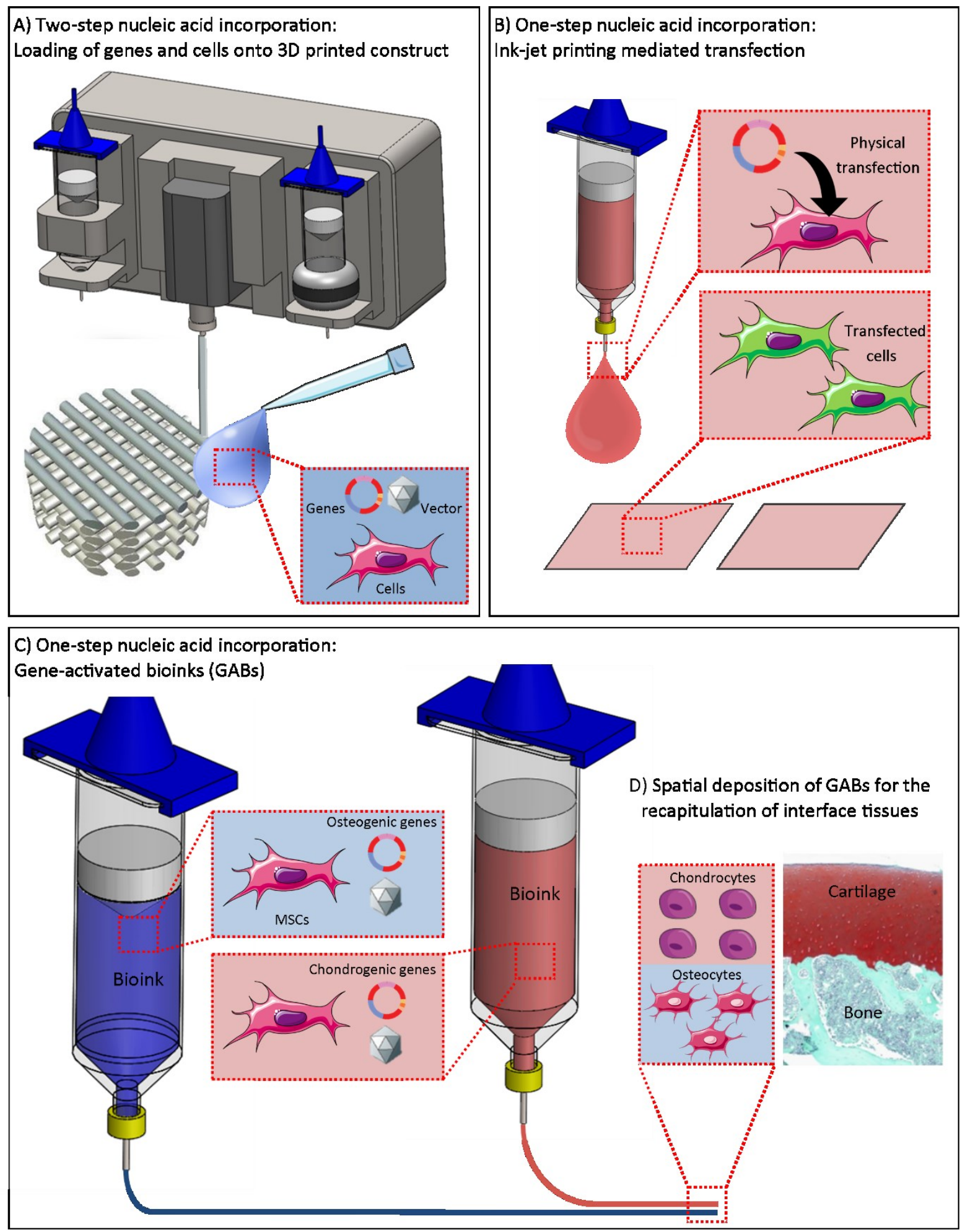

Figure 5. Nucleic acid incorporation into 3D bioprinting. (A) Two-step incorporation of cells and nucleic acids into porous 3D printed constructs by direct loading. (B) One-step incorporation of nucleic acids into the 3D printing process through the introduction of the genetic material into the cell population due to the forces applied during inkjet printing. (C) One-step incorporation 
of nucleic acids into the 3D printing process through the development of gene activated bioinks (GABs). (D) Recapitulation of the osteochondral architecture through the spatial deposition of chondrogenic and osteogenic GABs.

\subsubsection{Engineering Zonally Organised Interface Tissues Using Nucleic Acid Delivery}

The development of spatially-controlled gene delivery systems in combination with biofabrication techniques could also offer a very tunable strategy for interface TE. The cellmediated expression of the gene of interest could generate precisely controlled gradients of the gene product to enhance local cell differentiation or maintenance of a desired phenotype (Fig 5d), avoiding the use of supra-physiological concentrations of recombinant growth factors and associated side-effects ${ }^{212}$. Bi-phasic non-viral gene delivery in an oligo poly(ethylene glycol) fumarate (OPF) scaffold loaded with pDNA encoding for RUNX2 in the osteo layer and for the SOX trio in the chondro layer, showed increased subchondral bone formation in the osteo layer but failed to develop a well-integrated cartilage layer in an rat osteochondral defect model 213. Spatial distribution of pDNA encoding for either TGF- $\beta 1$ or BMP-2 in a MSC-laden, multiphasic collagen-based scaffold showed simultaneous cartilage and subchondral bone regeneration in a rabbit osteochondral defect ${ }^{15}$. Although the described studies highlight the potential of spatial gene delivery for the regeneration of complex interface tissues, 3D bioprinting might solve the limitations of traditional tissue engineering associated with poor layer integration, the scalability of the approach and the tissue organization present in the repair tissue. In addition, more sophisticated patterns of genetic material could be achieved using bioprinting technology in order to recapitulate the body's natural developmental and regenerative pathways. 


\section{Conclusions}

It is clear that 3D bioprinting holds tremendous promise for the future of cartilage and osteochondral tissue engineering. The adoption of the technology has already made it possible to engineer implants with increasing degrees of sophistication that better mimic the complexity of native cartilage and bone tissues. While significant progress has been made, a number of major challenges still exist, and it looks increasingly likely that the technology will now play a key role in addressing these challenges. 


\section{References}

1. Zhang, Y. \& Jordan, J. M. Epidemiology of osteoarthritis. Clin. Geriatr. Med. 26, 355-69 (2010).

2. Buckwalter, J. A., Saltzman, C. \& Brown, T. The impact of osteoarthritis: implications for research. Clin. Orthop. Relat. Res. S6-15 (2004).

3. Seil, R. \& Pape, D. Causes of failure and etiology of painful primary total knee arthroplasty. Knee Surg. Sports Traumatol. Arthrosc. 19, 1418-32 (2011).

4. Kerin, C., Malhotra, A., Spencer-Jones, R. \& Cool, W. P. When Do Total Knee Replacements Fail? J. Long. Term. Eff. Med. Implants 21, 51-54 (2011).

5. Labek, G., Thaler, M., Janda, W., Agreiter, M. \& Stockl, B. Revision rates after total joint replacement: CUMULATIVE RESULTS FROM WORLDWIDE JOINT REGISTER DATASETS. Bone Joint J. 93-B, 293-297 (2011).

6. Huey, D. J., Hu, J. C. \& Athanasiou, K. A. Unlike Bone, Cartilage Regeneration Remains Elusive. Science (80-. ). 338, 917-921 (2012).

7. Makris, E. a., Gomoll, A. H., Malizos, K. N., Hu, J. C. \& Athanasiou, K. a. Repair and tissue engineering techniques for articular cartilage. Nat. Rev. Rheumatol. 11, 21-34 (2014).

8. Keeney, M., Lai, J. H. \& Yang, F. Recent progress in cartilage tissue engineering. Curr. Opin. Biotechnol. 22, 734-740 (2011).

9. Yang, J., Shrike Zhang, Y., Yue, K. \& Khademhosseini, A. Cell-Laden Hydrogels for Osteochondral and Cartilage Tissue Engineering. Acta Biomater. (2017). doi:10.1016/j.actbio.2017.01.036 
10. Amini, A. R., Laurencin, C. T. \& Nukavarapu, S. P. Bone tissue engineering: recent advances and challenges. Crit. Rev. Biomed. Eng. 40, 363-408 (2012).

11. Bose, S., Roy, M. \& Bandyopadhyay, A. Recent advances in bone tissue engineering scaffolds. Trends Biotechnol. 30, 546-554 (2012).

12. Madry, H. et al. Enhanced repair of articular cartilage defects in vivo by transplanted chondrocytes overexpressing insulin-like growth factor I (IGF-I). Gene Ther. 12, $1171-1179$ (2005).

13. Wang, W. et al. The restoration of full-thickness cartilage defects with BMSCs and TGF-beta 1 loaded PLGA/fibrin gel constructs. Biomaterials 31, 8964-8973 (2010).

14. Shao, X. X., Hutmacher, D. W., Ho, S. T., Goh, J. C. H. \& Lee, E. H. Evaluation of a hybrid scaffold/cell construct in repair of high-load-bearing osteochondral defects in rabbits. Biomaterials 27, 1071-1080 (2006).

15. Chen, J. et al. Simultaneous regeneration of articular cartilage and subchondral bone in vivo using MSCs induced by a spatially controlled gene delivery system in bilayered integrated scaffolds. Biomaterials 32, 4793-4805 (2011).

16. Levingstone, T. J. et al. Multi-layered collagen-based scaffolds for osteochondral defect repair in rabbits. Acta Biomater. 32, 149-160 (2016).

17. Levingstone, T. J. et al. Cell-free multi-layered collagen-based scaffolds demonstrate layer specific regeneration of functional osteochondral tissue in caprine joints. Biomaterials 87, 69-81 (2016).

18. Kim, I. G., Ko, J., Lee, H. R., Do, S. H. \& Park, K. Mesenchymal cells condensationinducible mesh scaffolds for cartilage tissue engineering. Biomaterials 85, 18-29 (2016) 
19. Huang, B. J., Hu, J. C. \& Athanasiou, K. A. Cell-based tissue engineering strategies used in the clinical repair of articular cartilage. Biomaterials 98, 1-22 (2016).

20. Benninghoff, A. Form und Bau der Gelenkknorpel in Ihren Beziehungen zur Funktion. Z Zellforsch 2, 783-862 (1925).

21. Schuurman, W. et al. Cartilage regeneration using zonal chondrocyte subpopulations: a promising approach or an overcomplicated strategy? J. Tissue Eng. Regen. Med. 9, 669-678 (2015).

22. Buckwater, J. . \& Mankin, H. . Articular cartilage: tissue design and chondrocytematrix interactions. Instr. Course Lect. 47, 477-486 (1998).

23. Gannon, A. R., Nagel, T., Bell, A. P., Avery, N. C. \& Kelly, D. J. Postnatal Changes To the Mechanical Properties of Articular Cartilage Are Driven By the Evolution of Its Collagen Network. Eur. Cell. Mater. 29, 105-123 (2015).

24. Gannon, a R., Nagel, T. \& Kelly, D. J. The role of the superficial region in determining the dynamic properties of articular cartilage. Osteoarthritis Cartilage 20, $1417-25$ (2012).

25. Schinagl, R. M., Gurskis, D., Chen, A. C. \& Sah, R. L. Depth-dependent confined compression modulus of full-thickness bovine articular cartilage. J. Orthop. Res. 15, 499-506 (1997).

26. Chen, A. C., Bae, W. C., Schinagl, R. M. \& Sah, R. L. Depth and strain dependent mechanical and electromechanical properties of full-thickness bovine articular cartilage in confined compression. J. Biomech. 34, 1-12 (2001).

27. Kempson, G. E., Muir, H., Pollard, C. \& Tuke, M. The tensile properties of the cartilage of human femoral condyles related to the content of collagen and 
glycosaminoglycans. Biochim. Biophys. Acta - Gen. Subj. 297, 456-472 (1973).

28. Huey, D. J., Hu, J. C. \& Athanasiou, K. A. Unlike Bone, Cartilage Regeneration Remains Elusive. Science (80-. ). 338, (2012).

29. Mouser, V. H. M. et al. Three-Dimensional Bioprinting and Its Potential in the Field of Articular Cartilage Regeneration. Cartilage 1947603516665445 (2016). doi:10.1177/1947603516665445

30. Kang, H.-W., Yoo, J. J. \& Atala, A. in Methods in molecular biology (Clifton, N.J.) 1340, 161-169 (2015).

31. Murphy, S. V \& Atala, A. 3D bioprinting of tissues and organs. Nat. Biotechnol. 32, $773-785$ (2014).

32. Schuurman, W. et al. Bioprinting of hybrid tissue constructs with tailorable mechanical properties. Biofabrication 3, 21001 (2011).

33. Visser, J. et al. Reinforcement of hydrogels using three-dimensionally printed microfibres. Nat. Commun. 6, 6933 (2015).

34. Ren, X. et al. Engineering zonal cartilage through bioprinting collagen type II hydrogel constructs with biomimetic chondrocyte density gradient. $B M C$ Musculoskelet. Disord. 17, 301 (2016).

35. Pescosolido, L. et al. Hyaluronic acid and dextran-based semi-IPN hydrogels as biomaterials for bioprinting. Biomacromolecules 12, 1831-1838 (2011).

36. Schuurman, W. et al. Gelatin-methacrylamide hydrogels as potential biomaterials for fabrication of tissue-engineered cartilage constructs. Macromol. Biosci. 13, 551-61 (2013). 
37. Derby, B. et al. Bioprinting: inkjet printing proteins and hybrid cell-containing materials and structures. J. Mater. Chem. 18, 5717 (2008).

38. Nakamura, M. et al. Biocompatible Inkjet Printing Technique for Designed Seeding of Individual Living Cells. Tissue Eng. 11, 1658-1666 (2005).

39. Phillippi, J. a et al. Microenvironments engineered by inkjet bioprinting spatially direct adult stem cells toward muscle- and bone-like subpopulations. Stem Cells 26, 127-134 (2008).

40. Boland, T. et al. Drop-on-demand printing of cells and materials for designer tissue constructs. Mater. Sci. Eng. C 27, 372-376 (2007).

41. Faulkner-Jones, A. et al. Development of a valve-based cell printer for the formation of human embryonic stem cell spheroid aggregates. Biofabrication 5, 15013 (2013).

42. Lee, W. et al. Multi-layered culture of human skin fibroblasts and keratinocytes through three-dimensional freeform fabrication. Biomaterials 30, 1587-1595 (2009).

43. Fang, Y. et al. Rapid Generation of Multiplexed Cell Cocultures Using Acoustic Droplet Ejection Followed by Aqueous Two-Phase Exclusion Patterning. Tissue Eng. Part C Methods 18, 647-657 (2012).

44. Demirci, U. \& Montesano, G. Single cell epitaxy by acoustic picolitre droplets. Lab Chip 7, 1139-45 (2007).

45. Tasoglu, S. \& Demirci, U. Bioprinting for stem cell research. Trends Biotechnol. 31, 10-9 (2013).

46. Cui, X., Breitenkamp, K., Lotz, M. \& D'Lima, D. Synergistic action of fibroblast growth factor-2 and transforming growth factor-beta1 enhances bioprinted human neocartilage formation. Biotechnol. Bioeng. 109, 2357-2368 (2012). 
47. Cui, X., Breitenkamp, K., Finn, M. G., Lotz, M. \& D’Lima, D. D. Direct Human Cartilage Repair Using Three-Dimensional Bioprinting Technology. Tissue Eng. Part A 18, 1304-1312 (2012).

48. Ozbolat, I. T. \& Hospodiuk, M. Current advances and future perspectives in extrusionbased bioprinting. Biomaterials 76, 321-343 (2016).

49. Gudupati, H., Dey, M. \& Ozbolat, I. A Comprehensive Review on Droplet-based Bioprinting: Past, Present and Future. Biomaterials 102, 20-42 (2016).

50. Jia, J. et al. Engineering alginate as bioink for bioprinting. Acta Biomater. 10, 4323$4331(2014)$.

51. Kundu, J., Shim, J.-H., Jang, J., Kim, S.-W. \& Cho, D.-W. An additive manufacturingbased PCL-alginate-chondrocyte bioprinted scaffold for cartilage tissue engineering. $J$. Tissue Eng. Regen. Med. 9, 1286-97 (2015).

52. Poldervaart, M. T. et al. Sustained release of BMP-2 in bioprinted alginate for osteogenicity in mice and rats. PLoS One 8, e 72610 (2013).

53. Daly, A. C. et al. 3D Bioprinting of Developmentally Inspired Templates for Whole Bone Organ Engineering. Adv. Healthc. Mater. 5, 2305-2449 (2016).

54. Billiet, T., Gevaert, E., De Schryver, T., Cornelissen, M. \& Dubruel, P. The 3D printing of gelatin methacrylamide cell-laden tissue-engineered constructs with high cell viability. Biomaterials 35, 49-62 (2014).

55. Bertassonia, L. E. et al. Direct-write Bioprinting of Cell-laden Methacrylated Gelatin Hydrogels. Biofabrication 141, 520-529 (2008).

56. Fedorovich, N. E., De Wijn, J. R., Verbout, A. J., Alblas, J. \& Dhert, W. J. a. Threedimensional fiber deposition of cell-laden, viable, patterned constructs for bone tissue 
printing. Tissue Eng. Part A 14, 127-33 (2008).

57. Blaeser, A. et al. Biofabrication Under Fluorocarbon: A Novel Freeform Fabrication Technique to Generate High Aspect Ratio Tissue-Engineered Constructs. Biores. Open Access 2, 374-384 (2013).

58. Rhee, S., Puetzer, J. L., Mason, B. N., Reinhart-King, C. A. \& Bonassar, L. J. 3D Bioprinting of Spatially Heterogeneous Collagen Constructs for Cartilage Tissue Engineering. ACS Biomater. Sci. Eng. acsbiomaterials.6b00288 (2016). doi:10.1021/acsbiomaterials.6b00288

59. Yeo, M. G., Lee, J. S., Chun, W. \& Kim, G. H. An Innovative Collagen-Based CellPrinting Method for Obtaining Human Adipose Stem Cell-Laden Structures Consisting of Core-Sheath Structures for Tissue Engineering. Biomacromolecules 17, 1365-1375 (2016).

60. Wei Xu, W. et al. Rapid Prototyping Three-Dimensional Cell/Gelatin/Fibrinogen Constructs for Medical Regeneration. J. Bioact. Compat. Polym. 22, 363-377 (2007).

61. Murphy, S. V, Skardal, A. \& Atala, A. Evaluation of hydrogels for bio-printing applications. J. Biomed. Mater. Res. A 101A, 272-284 (2012).

62. Kang, H.-W. et al. A 3D bioprinting system to produce human-scale tissue constructs with structural integrity. Nat. Biotechnol. (2016). doi:10.1038/nbt.3413

63. Das, S. et al. Bioprintable, cell-laden silk fibroin-gelatin hydrogel supporting multilineage differentiation of stem cells for fabrication of three-dimensional tissue constructs. Acta Biomater. 11, 233-246 (2015).

64. Schacht, K. et al. Biofabrication of cell-loaded 3D spider silk constructs. Angew. Chemie - Int. Ed. 54, 2816-2820 (2015). 
65. Rodriguez, M. J. et al. Silk based bioinks for soft tissue reconstruction using 3dimensional (3D) printing with in vitro and in vivo assessments. Biomaterials 117, $105-115(2016)$.

66. Gao, G. et al. Improved properties of bone and cartilage tissue from 3D inkjetbioprinted human mesenchymal stem cells by simultaneous deposition and photocrosslinking in PEG-GelMA. Biotechnol. Lett. 37, 2349-2355 (2015).

67. Censi, R. et al. A printable photopolymerizable thermosensitive p(HPMAm-lactate)PEG hydrogel for tissue engineering. Adv. Funct. Mater. 21, 1833-1842 (2011).

68. Jia, W. et al. Direct 3D bioprinting of perfusable vascular constructs using a blend bioink. Biomaterials 106, 58-68 (2016).

69. Ouyang, L., Highley, C. B., Sun, W. \& Burdick, J. A. A Generalizable Strategy for the 3D Bioprinting of Hydrogels from Nonviscous Photo-crosslinkable Inks. Adv. Mater. 29, 1604983 (2017).

70. Highley, C. B., Rodell, C. B. \& Burdick, J. A. Direct 3D Printing of Shear-Thinning Hydrogels into Self-Healing Hydrogels. Adv. Mater. 27, 5075-5079 (2015).

71. Erickson, I. E. et al. Macromer density influences mesenchymal stem cell chondrogenesis and maturation in photocrosslinked hyaluronic acid hydrogels. Osteoarthr. Cartil. 17, 1639-1648 (2009).

72. Bian, L. et al. The influence of hyaluronic acid hydrogel crosslinking density and macromolecular diffusivity on human MSC chondrogenesis and hypertrophy. Biomaterials 34, 413-21 (2013).

73. Steward, A. J., Wagner, D. R. \& Kelly, D. J. The pericellular environment regulates cytoskeletal development and the differentiation of mesenchymal stem cells and 
determines their response to hydrostatic pressure. Eur. Cells Mater. 25, 167-178 (2013)

74. Malda, J. et al. 25th anniversary article: Engineering hydrogels for biofabrication. Adv. Mater. 25, 5011-28 (2013).

75. Blaeser, A. et al. Controlling Shear Stress in 3D Bioprinting is a Key Factor to Balance Printing Resolution and Stem Cell Integrity. Adv. Healthc. Mater. 326-333 (2015). doi:10.1002/adhm.201500677

76. Billiet, T., Vandenhaute, M., Van Vlierberghe, S., Schelfhout, J. \& Dubruel, P. A review of trends and limitations in hydrogel-rapid prototyping for tissue engineering. Biomaterials 33, 6020-6041 (2012).

77. Nair, K. et al. Characterization of cell viability during bioprinting processes. Biotechnol. J. 4, 1168-1177 (2009).

78. Melchels, F. P. W., Dhert, W. J. a., Hutmacher, D. W. \& Malda, J. Development and characterisation of a new bioink for additive tissue manufacturing. J. Mater. Chem. B 2, $2282(2014)$.

79. Skardal, A. \& Atala, A. Biomaterials for Integration with 3-D Bioprinting. Ann. Biomed. Eng. 43, 730-746 (2015).

80. Jungst, T., Smolan, W., Schacht, K., Scheibel, T. \& Groll, J. Strategies and Molecular Design Criteria for 3D Printable Hydrogels. Chem. Rev. 116, 1496-1539 (2016).

81. Schuurman, W. et al. Biofabrication of Osteochondral Tissue Equivalents by Printing Topologically Defined, Cell-Laden Hydrogel Scaffolds. Tissue Eng. Part C Methods 18, 33-44 (2012).

82. Costantini, M. et al. 3D bioprinting of BM-MSCs-loaded ECM biomimetic hydrogels 
for in vitro neocartilage formation. Biofabrication 8, 35002 (2016).

83. Daly, A. C., Critchley, S. E., Rensock, E. M. \& Kelly, D. J. A Comparison of Different Bioinks for 3D Bioprinting of Fibrocartilage and Hyaline Cartilage. Biofabrication 8, $1-25(2016)$.

84. Levett, P. a et al. A biomimetic extracellular matrix for cartilage tissue engineering centered on photocurable gelatin, hyaluronic acid and chondroitin sulfate. Acta Biomater. 10, 214-23 (2014).

85. Abbadessa, A. et al. A Synthetic Thermosensitive Hydrogel for Cartilage Bioprinting and Its Biofunctionalization with Polysaccharides. Biomacromolecules 17, 2137-2147 (2016).

86. Markstedt, K. et al. 3D bioprinting human chondrocytes with nanocellulose-alginate bioink for cartilage tissue engineering applications. Biomacromolecules 16, 1489-1496 (2015).

87. Müller, M., Ozturk, E., Arlov, O., Gatenholm, P. \& Zenobi-Wong, M. Alginate sulfate-nanocellulose bioinks for cartilage bioprinting applications. Ann. Biomed. Eng. (2016). doi:10.1007/s10439-016-1704-5

88. Öztürk, E. et al. Sulfated Hydrogel Matrices Direct Mitogenicity and Maintenance of Chondrocyte Phenotype through Activation of FGF Signaling. Adv. Funct. Mater. 26, 3649-3662 (2016).

89. Pati, F. et al. Printing three-dimensional tissue analogues with decellularized extracellular matrix bioink. Nat. Commun. 5, 3935 (2014).

90. Choi, Y. J. et al. 3D Cell Printing of Functional Skeletal Muscle Constructs Using Skeletal Muscle-Derived Bioink. Adv. Healthc. Mater. 2636-2645 (2016). 
doi:10.1002/adhm.201600483

91. Kesti, M. et al. Bioprinting Complex Cartilaginous Structures with Clinically Compliant Biomaterials. Adv. Funct. Mater. $7406-7417$ (2015).

doi:10.1002/adfm.201503423

92. Kesti, M. et al. A versatile bioink for three-dimensional printing of cellular scaffolds based on thermally and photo-triggered tandem gelation. Acta Biomater. 11, 162-72 (2015).

93. Yu, Y. et al. Three-dimensional bioprinting using self-assembling scalable scaffoldfree 'tissue strands' as a new bioink. Sci. Rep. 6, 28714 (2016).

94. Armstrong, J. P. K., Burke, M., Carter, B. M., Davis, S. A. \& Perriman, A. W. 3D Bioprinting Using a Templated Porous Bioink. Adv. Healthc. Mater. 5, 1724-1730 (2016).

95. Ahn, S., Lee, H., Bonassar, L. J. \& Kim, G. Cells (MC3T3-E1)-Laden Alginate Scaffolds Fabricated by a Modified Solid-Freeform Fabrication Process Supplemented with an Aerosol Spraying. Biomacromolecules 13, 2997-3003 (2012).

96. Mouser, V. H. M. et al. Yield stress determines bioprintability of hydrogels based on gelatin-methacryloyl and gellan gum for cartilage bioprinting. Biofabrication $\mathbf{8}, 1-13$ (2016).

97. Park, J. Y. et al. A comparative study on collagen type I and hyaluronic acid dependent cell behavior for osteochondral tissue bioprinting. Biofabrication 6, 35004 (2014)

98. $\mathrm{Xu}, \mathrm{T}$. et al. Hybrid printing of mechanically and biologically improved constructs for cartilage tissue engineering applications. Biofabrication 5, 15001 (2013). 
99. Kang, H.-W. et al. A 3D bioprinting system to produce human-scale tissue constructs with structural integrity. Nat. Biotechnol. 34, 312-319 (2016).

100. Cui, X., Breitenkamp, K., Finn, M. G., Lotz, M. \& D’Lima, D. Direct Human Cartilage Repair Using 3D Bioprinting Technology. Tissue Eng. 18, 1304-1312 (2012).

101. Cohen, D. L., Malone, E., Lipson, H. \& Bonassar, L. J. Direct freeform fabrication of seeded hydrogels in arbitrary geometries. Tissue Eng. 12, 1325-35 (2006).

102. Fedorovich, N. E. et al. Hydrogels as extracellular matrices for skeletal tissue engineering: state-of-the-art and novel application in organ printing. Tissue Eng. 13, 1905-1925 (2007).

103. Fedorovich, N. E., Kuipers, E., Gawlitta, D., Dhert, W. J. a. \& Alblas, J. Scaffold Porosity and Oxygenation of Printed Hydrogel Constructs Affect Functionality of Embedded Osteogenic Progenitors. Tissue Eng. Part A 17, 2473-2486 (2011).

104. Poldervaart, M. T. et al. Sustained release of BMP-2 in bioprinted alginate for osteogenicity in mice and rats. PLoS One 8, e 72610 (2013).

105. Cunniffe, G. M. et al. Chondrogenically primed mesenchymal stem cell-seeded alginate hydrogels promote early bone formation in critically-sized defects. Eur. Polym. J. 72, 464-472 (2015).

106. Simmons, C. a, Alsberg, E., Hsiong, S., Kim, W. J. \& Mooney, D. J. Dual growth factor delivery and controlled scaffold degradation enhance in vivo bone formation by transplanted bone marrow stromal cells. Bone 35, 562-9 (2004).

107. Alsberg, E., Anderson, K. W., Albeiruti, A., Rowley, J. A. \& Mooney, D. J. Engineering growing tissues. PNAS 99, 12025-12030 (2002). 
108. Alsberg, E. et al. Regulating bone formation via controlled scaffold degradation. $J$. Dent. Res. 82, 903-8 (2003).

109. Wang, X.-F. et al. Osteogenic Differentiation of Three-Dimensional Bioprinted Constructs Consisting of Human Adipose-Derived Stem Cells In Vitro and In Vivo. PLoS One 11, e0157214 (2016).

110. Du, M. et al. 3D bioprinting of BMSC-laden methacrylamide gelatin scaffolds with CBD-BMP2-collagen microfibers. Biofabrication 7, 44104 (2015).

111. Duarte Campos, D. F. et al. Bioprinting Organotypic Hydrogels with Improved Mesenchymal Stem Cell Remodeling and Mineralization Properties for Bone Tissue Engineering. Adv. Healthc. Mater. 5, 1336-1345 (2016).

112. Wang, X. et al. Effect of bioglass on growth and biomineralization of saos-2 cells in hydrogel after 3d cell bioprinting. PLoS One 9, 1-7 (2014).

113. Luo, Y., Wu, C., Lode, A. \& Gelinsky, M. Hierarchical mesoporous bioactive glass/alginate composite scaffolds fabricated by three-dimensional plotting for bone tissue engineering. Biofabrication 5, 15005-15018 (2013).

114. Martin, Y., Eldardiri, M., Lawrence-Watt, D. J. \& Sharpe, J. R. Microcarriers and Their Potential in Tissue Regeneration. Tissue Eng. Part B 17, 71-80 (2011).

115. Sart, S., Agathos, S. N. \& Li, Y. Engineering stem cell fate with biochemical and biomechanical properties of microcarriers. Biotechnol. Prog. 29, 1354-1366 (2013).

116. Levato, R. et al. Biofabrication of tissue constructs by 3D bioprinting of cell-laden microcarriers. Biofabrication 6, 35020 (2014).

117. Wüst, S., Godla, M. E., Müller, R. \& Hofmann, S. Tunable hydrogel composite with two-step processing in combination with innovative hardware upgrade for cell-based 
three-dimensional bioprinting. Acta Biomater. 10, 630-40 (2014).

118. Martinez-Vazquez, F. J., Cabanas, M. V., Paris, J. L., Lozano, D. \& Vallet-Regi, M. Fabrication of novel Si-doped hydroxyapatite/gelatine scaffolds by rapid prototyping for drug delivery and bone regeneration. Acta Biomater. 15, 200-209 (2015).

119. Sultana, N. \& Wang, M. Fabrication of HA/PHBV composite scaffolds through the emulsion freezing/freeze-drying process and characterisation of the scaffolds. J. Mater. Sci. Mater. Med. 19, 2555-2561 (2008).

120. Sawkins, M. J. et al. Cell and protein compatible 3D bioprinting of mechanically strong constructs for bone repair. Biofabrication 7, 35004 (2015).

121. Zigang Ge et al. Proliferation and differentiation of human osteoblasts within 3D printed poly-lactic-co-glycolic acid scaffolds. J. Biomater. Appl. 23, 533-547 (2009).

122. Holmes, B., Bulusu, K. \& Plesniak, M. A synergistic approach to the design, fabrication and evaluation of 3D printed micro and nano featured scaffolds for vascularized bone tissue repair. Nanotechnology 27, 64001 (2016).

123. Lee, S. J. et al. Surface modification of 3D-printed porous scaffolds via musselinspired polydopamine and effective immobilization of rhBMP-2 to promote osteogenic differentiation for bone tissue engineering. Acta Biomater. 40, 182-191 (2016).

124. Shim, J.-H. et al. Efficacy of rhBMP-2 loaded PCL/PLGA/ $\beta$-TCP guided bone regeneration membrane fabricated by $3 \mathrm{D}$ printing technology for reconstruction of calvaria defects in rabbit. Biomed. Mater. 9, 65006 (2014).

125. Zysset, P. K., Edward Guo, X., Edward Hoffler, C., Moore, K. E. \& Goldstein, S. A. Elastic modulus and hardness of cortical and trabecular bone lamellae measured by 
nanoindentation in the human femur. J. Biomech. 32, 1005-1012 (1999).

126. Rho, J. Y. et al. Mechanical properties and the hierarchical structure of bone. Med. Eng. Phys. 20, 92-102 (1998).

127. Mercado-Pagán, Á. E., Stahl, A. M., Shanjani, Y. \& Yang, Y. Vascularization in Bone Tissue Engineering Constructs. Ann. Biomed. Eng. 43, 718-729 (2015).

128. Kang, H.-W. et al. A 3D bioprinting system to produce human-scale tissue constructs with structural integrity. Nat. Biotechnol. 3-7 (2016). doi:10.1038/nbt.3413

129. Cui, H. et al. Hierarchical Fabrication of Engineered Vascularized Bone Biphasic Constructs via Dual 3D Bioprinting: Integrating Regional Bioactive Factors into Architectural Design. Adv. Healthc. Mater. 1-8 (2016). doi:10.1002/adhm.201600505

130. Poldervaart, M. T. et al. Prolonged presence of VEGF promotes vascularization in 3D bioprinted scaffolds with defined architecture. J. Control. Release 184, 58-66 (2014).

131. Park, J. Y. et al. 3D printing technology to control BMP-2 and VEGF delivery spatially and temporally to promote large-volume bone regeneration. J. Mater. Chem. B 3, 5415-5425 (2015).

132. Boere, K. W. M. et al. Covalent attachment of a three-dimensionally printed thermoplast to a gelatin hydrogel for mechanically enhanced cartilage constructs. Acta Biomater. 10, 2602-2611 (2014).

133. Sachlos, E. \& Czernuszka, J. T. Making tissue engineering scaffolds work. Review on the application of solid freeform fabrication technology to the production of tissue engineering scaffolds. Eur. Cells Mater. 5, 29-40 (2003).

134. Zein, I., Hutmacher, D. W., Tan, K. C. \& Teoh, S. H. Fused deposition modeling of novel scaffold architectures for tissue engineering applications. Biomaterials 23, 
1169-1185 (2002).

135. Olubamiji, A. D. et al. Modulating mechanical behaviour of 3D-printed cartilagemimetic PCL scaffolds: influence of molecular weight and pore geometry. Biofabrication 8, 25020 (2016).

136. Hutmacher, D. W. et al. Mechanical properties and cell cultural response of polycaprolactone scaffolds designed and fabricated via fused deposition modeling. $J$. Biomed. Mater. Res. 55, 203-216 (2001).

137. Goldstein, S. a. The mechanical properties of trabecular bone: dependence on anatomic location and function. J. Biomech. 20, 1055-1061 (1987).

138. Chen, S. S., Falcovitz, Y. H., Schneiderman, R., Maroudas, a. \& Sah, R. L. Depthdependent compressive properties of normal aged human femoral head articular cartilage: Relationship to fixed charge density. Osteoarthr. Cartil. 9, 561-569 (2001).

139. Woodfield, T. B. F. et al. Design of porous scaffolds for cartilage tissue engineering using a three-dimensional fiber-deposition technique. Biomaterials 25, 4149-4161 (2004).

140. Malda, J. et al. The effect of PEGT/PBT scaffold architecture on oxygen gradients in tissue engineered cartilaginous constructs. Biomaterials 25, 5773-80 (2004).

141. Swieszkowski, W., Tuan, B. H. S., Kurzydlowski, K. J. \& Hutmacher, D. W. Repair and regeneration of osteochondral defects in the articular joints. Biomol. Eng. 24, 489495 (2007).

142. Barron, V. et al. Evaluation of Cartilage Repair by Mesenchymal Stem Cells Seeded on a PEOT/PBT Scaffold in an Osteochondral Defect. Ann. Biomed. Eng. 43, 2069$2082(2015)$ 
143. Di Luca, A. et al. Gradients in pore size enhance the osteogenic differentiation of human mesenchymal stromal cells in three-dimensional scaffolds. Sci. Rep. 6, 22898 (2016).

144. Di Luca, A. et al. Influencing chondrogenic differentiation of human mesenchymal stromal cells in scaffolds displaying a structural gradient in pore size. Acta Biomater. 36, 210-219 (2016).

145. Di Luca, A. et al. Surface energy and stiffness discrete gradients in additive manufactured scaffolds for osteochondral regeneration. Biofabrication 8, 15014 (2016).

146. Kim, G. H. \& Son, J. G. 3D polycarprolactone (PCL) scaffold with hierarchical structure fabricated by a piezoelectric transducer (PZT)-assisted bioplotter. Appl. Phys. A Mater. Sci. Process. 94, 781-785 (2009).

147. Seyednejad, H. et al. In vivo biocompatibility and biodegradation of 3D-printed porous scaffolds based on a hydroxyl-functionalized poly( $\varepsilon$-caprolactone). Biomaterials 33, 4309-4318 (2012).

148. Park, S. H. et al. Scaffolds for bone tissue engineering fabricated from two different materials by the rapid prototyping technique: PCL versus PLGA. J. Mater. Sci. Mater. Med. 23, 2671-2678 (2012).

149. Park, S. H. et al. Scaffolds for bone tissue engineering fabricated from two different materials by the rapid prototyping technique: PCL versus PLGA. J. Mater. Sci. Mater. Med. 23, 2671-2678 (2012).

150. Heo, S. J. et al. In vitro and animal study of novel nano-hydroxyapatite/poly(ÎI caprolactone) composite scaffolds fabricated by layer manufacturing process. Tissue 
Eng. - Part A 15, 977-989 (2009).

151. Kim, J. et al. Rapid-prototyped PLGA/ $\beta$-TCP/hydroxyapatite nanocomposite scaffolds in a rabbit femoral defect model. Biofabrication 4, 25003 (2012).

152. Reichert, J. C. et al. Custom-made composite scaffolds for segmental defect repair in long bones. Int. Orthop. 35, 1229-1236 (2011).

153. Lee, J.-S. et al. 3D printing of composite tissue with complex shape applied to ear regeneration. Biofabrication 6, 24103 (2014).

154. Shim, J.-H. et al. Three-dimensional bioprinting of multilayered constructs containing human mesenchymal stromal cells for osteochondral tissue regeneration in the rabbit knee joint. Biofabrication 8, 14102 (2016).

155. Mouser, V. H. M. et al. Development of a thermosensitive HAMA-containing bio-ink for the fabrication of composite cartilage repair constructs. Biofabrication 15026, (2017).

156. Lam, C. X. F., Savalani, M. M., Teoh, S.-H. \& Hutmacher, D. W. Dynamics of in vitro polymer degradation of polycaprolactone-based scaffolds: accelerated versus simulated physiological conditions. Biomed. Mater. 3, 34108 (2008).

157. Woodruff, M. A. \& Hutmacher, D. W. The return of a forgotten polymerPolycaprolactone in the 21st century. Prog. Polym. Sci. 35, 1217-1256 (2010).

158. Seyednejad, H. et al. Synthesis and Characterization of Hydroxyl-Functionalized Caprolactone Copolymers and Their Effect on Adhesion, Proliferation, and Differentiation of Human Mesenchymal Stem Cells. Biomacromolecules 10, 30483054 (2009).

159. Anderson, J. M. \& Shive, M. S. Biodegradation and biocompatibility of PLA and 
PLGA microspheres. Adv. Drug Deliv. Rev. 64, 72-82 (2012).

160. Fu, K., Pack, D. W., Klibanov, A. M. \& Langer, R. Visual evidence of acidic environment within degrading poly(lactic-co- glycolic acid) (PLGA) microspheres. Pharm. Res. 17, 100-106 (2000).

161. Lee, Y., Kwon, J., Khang, G. \& Lee, D. Reduction of inflammatory responses and enhancement of extracellular matrix formation by vanillin-incorporated poly(lactic-coglycolic acid) scaffolds. Tissue Eng. Part A 18, 1967-78 (2012).

162. Yang, S. S. et al. Fabrication of an osteochondral graft with using a solid freeform fabrication system. Tissue Eng. Regen. Med. 12, 239-248 (2015).

163. Hochleitner, G. et al. Additive manufacturing of scaffolds with sub-micron filaments via melt electrospinning writing. Biofabrication 7, 35002 (2015).

164. Brown, T. D., Dalton, P. D. \& Hutmacher, D. W. Direct writing by way of melt electrospinning. Adv. Mater. 23, 5651-7 (2011).

165. Hochleitner, G. et al. Fibre pulsing during melt electrospinning writing. BioNanoMaterials 0, 21-23 (2016).

166. Guilak, F., Ratcliffe, A. \& Mow, V. C. Chondrocyte deformation and local tissue strain in articular cartilage: A confocal microscopy study. J. Orthop. Res. 13, 410-421 (1995).

167. Bas, O. et al. Biofabricated soft network composites for cartilage tissue engineering. Biofabrication (2010). doi:10.1042/BJ20101136>

168. Ding, C. et al. Regeneration of a goat femoral head using a tissue-specific, biphasic scaffold fabricated with CAD/CAM technology. Biomaterials 34, 6706-16 (2013). 
169. Woodfield, T. B. F. et al. Rapid prototyping of anatomically shaped, tissue-engineered implants for restoring congruent articulating surfaces in small joints. Cell Prolif. 42, 485-497 (2009).

170. Lee, C. H. et al. Regeneration of the articular surface of the rabbit synovial joint by cell homing: a proof of concept study. Lancet 376, 440-448 (2010).

171. Tarafder, S. et al. Micro-precise spatiotemporal delivery system embedded in 3D printing for complex tissue regeneration. Biofabrication 8, 25003 (2016).

172. Woodfield, T. B. F. et al. Rapid prototyping of anatomically shaped, tissue-engineered implants for restoring congruent articulating surfaces in small joints. Cell Prolif. 42, 485-497 (2009).

173. Gharravi, A. M. et al. Design and fabrication of anatomical bioreactor systems containing alginate scaffolds for cartilage tissue engineering. Avicenna J. Med. Biotechnol. 4, 65-74 (2012).

174. Chen, Q., Chen, H., Zhu, L. \& Zheng, J. Materials Chemistry B Fundamentals of double network hydrogels. J. Mater. Chem. B 3, 3645-3886 (2015).

175. Gong, J. P. Why are double network hydrogels so tough? Soft Matter 6, 2583 (2010).

176. Hong, S. et al. 3D Printing: 3D Printing of Highly Stretchable and Tough Hydrogels into Complex, Cellularized Structures. Adv. Mater. 27, 4034 (2015).

177. Wang, X. et al. Growth factor gradients via microsphere delivery in biopolymer scaffolds for osteochondral tissue engineering. J. Control. Release 134, 81-90 (2009).

178. Albro, M. B. et al. Heterogeneous engineered cartilage growth results from gradients of media-supplemented active TGF- $\beta$ and is ameliorated by the alternative supplementation of latent TGF- $\beta$. Biomaterials 77, 173-185 (2016). 
179. Miller, E. D. et al. Inkjet printing of growth factor concentration gradients and combinatorial arrays immobilized on biologically-relevant substrates. Comb. Chem. High Throughput Screen. 12, 604-618 (2009).

180. Jeon, O., Alt, D. S., Linderman, S. W. \& Alsberg, E. Biochemical and physical signal gradients in hydrogels to control stem cell behavior. Adv. Mater. 25, 6366-72 (2013).

181. Moeinzadeh, S., Pajoum Shariati, S. R. \& Jabbari, E. Comparative Effect of Physicomechanical and Biomolecular Cues on Zone-Specific Chondrogenic Differentiation of Mesenchymal Stem Cells. Biomaterials 92, 57-70 (2016).

182. Liu, W. et al. Rapid Continuous Multimaterial Extrusion Bioprinting. Adv. Mater. 1604630, $1-8$ (2016).

183. Visser, J. et al. Biofabrication of multi-material anatomically shaped tissue constructs. Biofabrication 5, 35007 (2013).

184. Utzinger, U., Baggett, B., Weiss, J. A., Hoying, J. B. \& Edgar, L. T. Large-scale time series microscopy of neovessel growth during angiogenesis. Angiogenesis 18, 219-232 (2015).

185. Unger, R. E. et al. The rapid anastomosis between prevascularized networks on silk fibroin scaffolds generated in vitro with cocultures of human microvascular endothelial and osteoblast cells and the host vasculature. Biomaterials 31, 6959-6967 (2010).

186. Weinandy, S. et al. Biofunctionalized microfiber-assisted formation of intrinsic threedimensional capillary-like structures. Tissue Eng. Part A 20, 1858-69 (2014).

187. Chen, Y.-C. et al. Functional Human Vascular Network Generated in Photocrosslinkable Gelatin Methacrylate Hydrogels. Adv. Funct. Mater. 22, 2027 2039 (2012). 
188. Kang, Y., Mochizuki, N., Khademhosseini, A., Fukuda, J. \& Yang, Y. Engineering a vascularized collagen- $\hat{I}^{2}$-tricalcium phosphate graft using an electrochemical approach. Acta Biomater. 11, 449-458 (2015).

189. Miller, J. S. et al. Rapid casting of patterned vascular networks for perfusable engineered three-dimensional tissues. Nat. Mater. 11, 768-74 (2012).

190. Golden, A. P. \& Tien, J. Fabrication of microfluidic hydrogels using molded gelatin as a sacrificial element $\{$. doi:10.1039/b618409j

191. Lee, V. K. et al. Creating perfused functional vascular channels using 3D bio-printing technology. Biomaterials 35, 8092-8102 (2014).

192. Bertassoni, L. E. et al. Hydrogel bioprinted microchannel networks for vascularization of tissue engineering constructs. Lab Chip 14, 2202-2211 (2014).

193. Kolesky, D. B. et al. 3D Bioprinting of Vascularized, Heterogeneous Cell-Laden Tissue Constructs. Adv. Mater. 26, 3124-3130 (2014).

194. Kolesky, D. B., Homan, K. A., Skylar-Scott, M. A. \& Lewis, J. A. Three-dimensional bioprinting of thick vascularized tissues. Proc. Natl. Acad. Sci. U. S. A. 113, 3179-84 (2016).

195. Linville, R. M., Boland, N. F., Covarrubias, G., Price, G. M. \& Tien, J. Physical and Chemical Signals That Promote Vascularization of Capillary-Scale Channels. Cell. Mol. Bioeng. 9, 73-84 (2016).

196. Hammer, J., Han, L.-H., Tong, X. \& Yang, F. A facile method to fabricate hydrogels with microchannel-like porosity for tissue engineering. Tissue Eng. Part C. Methods 20, 169-76 (2014).

197. Lee, V. K. et al. Generation of multi-scale vascular network system within 3D 
hydrogel using 3D bio-printing technology. Cell. Mol. Bioeng. 7, 460-472 (2014).

198. Colosi, C. et al. Microfluidic Bioprinting of Heterogeneous 3D Tissue Constructs Using Low-Viscosity Bioink. Adv. Mater. 28, 677-684a (2016).

199. Vickerman, V., Blundo, J., Chung, S. \& Kamm, R. D. Design, fabrication and implementation of a novel multi-parameter control microfluidic platform for threedimensional cell culture and real-time imaging. Lab Chip 8, 1468-1477 (2008).

200. Chung, S. et al. Cell migration into scaffolds under co-culture conditions in a microfluidic platform. Lab Chip 9, 269-275 (2009).

201. Odedra, D., Chiu, L. L. Y., Shoichet, M. \& Radisic, M. Endothelial cells guided by immobilized gradients of vascular endothelial growth factor on porous collagen scaffolds. Acta Biomater. 7, 3027-35 (2011).

202. Guex, A. G. et al. Covalent immobilisation of VEGF on plasma-coated electrospun scaffolds for tissue engineering applications. Colloids Surf. B. Biointerfaces 123, 724 33 (2014).

203. Evans, C. Using genes to facilitate the endogenous repair and regeneration of orthopaedic tissues. Int. Orthop. 38, 1761-9 (2014).

204. Gonzalez-Fernandez, T., Tierney, E. G., Cunniffe, G. M., O’Brien, F. J. \& Kelly, D. J. Gene Delivery of TGF- $\beta 3$ and BMP2 in an MSC-Laden Alginate Hydrogel for Articular Cartilage and Endochondral Bone Tissue Engineering. Tissue Eng. Part A 22, 776-87 (2016).

205. Curtin, C. M. et al. Combinatorial gene therapy accelerates bone regeneration: nonviral dual delivery of VEGF and BMP2 in a collagen-nanohydroxyapatite scaffold. Adv. Healthc. Mater. 4, 223-7 (2015). 
206. Loozen, L. D., Wegman, F., Öner, F. C., Dhert, W. J. a. \& Alblas, J. Porous bioprinted constructs in BMP-2 non-viral gene therapy for bone tissue engineering. J. Mater. Chem. B 2, (2013).

207. Wegman, F. et al. Gene delivery of bone morphogenetic protein-2 plasmid DNA promotes bone formation in a large animal model. J. Tissue Eng. Regen. Med. 8, 763770 (2014).

208. Xu, T. et al. Inkjet-mediated gene transfection into living cells combined with targeted delivery. Tissue Eng. Part A 15, 95-101 (2009).

209. Cui, X., Dean, D., Ruggeri, Z. M. \& Boland, T. Cell damage evaluation of thermal inkjet printed Chinese hamster ovary cells. Biotechnol. Bioeng. 106, 963-969 (2010).

210. Loozen, L. D. et al. Bone Morphogenetic Protein-2 Nonviral Gene Therapy in a Goat Iliac Crest Model for Bone Formation. Tissue Eng. Part A 21, 150413075110008 (2015).

211. Brunger, J. M. et al. Scaffold-mediated lentiviral transduction for functional tissue engineering of cartilage. Proc. Natl. Acad. Sci. U. S. A. 111, E798-806 (2014).

212. Zara, J. N. et al. High doses of bone morphogenetic protein 2 induce structurally abnormal bone and inflammation in vivo. Tissue Eng. Part A 17, 1389-99 (2011).

213. Needham, C. J. et al. Osteochondral tissue regeneration through polymeric delivery of DNA encoding for the SOX trio and RUNX2. Acta Biomater. 10, 4103-4112 (2014). 